\title{
The liquid-hydrogen absorber for MICE
}

\author{
MICE Collaboration \\ V. Bayliss, J. Boehm, T. Bradshaw, M. Courthold, S. Harrison, \\ M. Hills, P. Hodgson, S. Ishimoto, A. Kurup, W. Lau, K. Long, \\ A. Nichols, D. Summers, M. Tucker, P. Warburton, \\ S. Watson and C. Whyte
}

\section{Published version information}

Citation: V Bayliss et al. "The liquid-hydrogen absorber for MICE." Journal of Instrumentation, vol. 13, no. 9 (2018): T09008.

DOI: $10.1088 / 1748-0221 / 13 / 09 / T 09008$

This is an author-created, un-copyedited version of an article accepted for publication in the Journal of Instrumentation. The publisher is not responsible for any errors or omissions in this version of the manuscript or any version derived from $i t$. The Version of Record is available online at DOI above.

This version is made available in accordance with publisher policies. Please cite only the published version using the reference above. This is the citation assigned by the publisher at the time of issuing the AAM/APV. Please check the publisher's website for any updates. 


\title{
The liquid-hydrogen absorber for MICE
}

\section{MICE collaboration}

\author{
V. Bayliss, ${ }^{a}$ J. Boehm, ${ }^{a}$ T. Bradshaw, ${ }^{a}$ M. Courthold, ${ }^{a}$ S. Harrison, ${ }^{a} \star^{\star}$ M. Hills, ${ }^{a}$ P. Hodgson, ${ }^{b}$ \\ S. Ishimoto, ${ }^{c}$ A. Kurup, ${ }^{d}$ W. Lau, ${ }^{e}$ K. Long, ${ }^{d, a}$ A. Nichols, ${ }^{a}$ D. Summers, ${ }^{f}$ M. Tucker, ${ }^{a, 1}$ \\ P. Warburton, ${ }^{g}$ S. Watson, ${ }^{a, \dagger}$ and C. Whyte ${ }^{h, i, j}$ \\ ${ }^{a}$ STFC Rutherford Appleton Laboratory, Harwell Oxford, Didcot, OX11 OQX, UK \\ ${ }^{b}$ Department of Physics and Astronomy, University of Sheffield, Sheffield, S3 7RH, UK \\ ${ }^{c}$ KEK, Oho 1-1, Tskuba, Ibaraki 305-0801, Japan \\ ${ }^{d}$ Department of Physics, Blackett Laboratory, Imperial College London, London, SW7 2BB, UK \\ ${ }^{e}$ Particle Physics Department, The Denys Wilkinson Building, Keble Road, Oxford OX1 3RH, UK \\ ${ }^{f}$ Department of Physics, University of Mississippi-Oxford, University, MS 38677, USA \\ ${ }^{g}$ STFC Daresbury Laboratory, Daresbury, Cheshire, WA4 4AD, UK \\ ${ }^{h}$ Department of Physics, University of Strathclyde, Glasgow, G1 1XJ, UK \\ ${ }^{i}$ SUPA, University of Glasgow, Glasgow, G12 8QQ, UK \\ ${ }^{j}$ Cockroft Institute, Keckwick Lane, Daresbury, Warrington, WA4 4AD, UK \\ ${ }^{\star}$ Now at: AS Scientific Products Ltd, 2 Barton Lane, Abingdon, OX14 3NB, UK \\ ${ }^{\dagger}$ Now at: Astronomy Technology Centre, Royal Observatory, Blackford Hill, Edinburgh, EH9 3HJ, UK \\ E-mail: mark.tucker@stfc.ukri.org
}

\begin{abstract}
The Muon Ionization Cooling Experiment (MICE) has been built at the STFC Rutherford Appleton Laboratory to demonstrate the principle of muon beam phase-space reduction via ionization cooling. Muon beam cooling will be required at a future proton-derived neutrino factory or muon collider. Ionization cooling is achieved by passing the beam through an energy-absorbing material, such as liquid hydrogen, and then re-accelerating the beam using RF cavities. This paper describes the hydrogen system constructed for MICE including: the liquid-hydrogen absorber, its associated cryogenic and gas systems, the control and monitoring system, and the necessary safety engineering. The performance of the system in cool-down, liquefaction, and stable operation is also presented.
\end{abstract}

Keywords: Accelerator subsystems and technologies, Beam optics, Gas systems and purification

\footnotetext{
${ }^{1}$ Corresponding author.
} 


\section{Contents}

1 Introduction 1

2 The Muon Ionization Cooling Experiment 2

2.1 Beamline 2

2.2 Safety considerations 3

2.3 Safety approval process 4

3 Absorber vessel 5

3.1 Design considerations 5

3.2 Absorber vessel body 6

3.3 Windows 8

$\begin{array}{lll}\text { 3.3.1 Window manufacture } & 8\end{array}$

3.3.2 Window thickness measurement 9

$\begin{array}{lll}\text { 3.3.3 Window burst tests } & 10\end{array}$

4 Condensing unit 11

5 Gas system 14

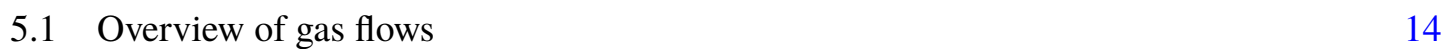

$\begin{array}{ll}\text { 5.2 Safety volume and quench line } & 16\end{array}$

$\begin{array}{lll}5.3 \text { Gas panel } & 17\end{array}$

6 Control and monitoring system 18

7 Performance $\quad 20$

$\begin{array}{lll}7.1 & \text { Initial tests with helium gas } & 21\end{array}$

$\begin{array}{ll}7.2 & \text { Liquefaction of neon } \\ \end{array}$

$\begin{array}{lll}\text { 7.2.1 Off-line } & 21\end{array}$

7.2.2 Within MICE 22

$\begin{array}{ll}7.3 & \text { Liquefaction of hydrogen } \\ & 22\end{array}$

8 Conclusions $\quad 24$

\section{Introduction}

Stored muon beams have been proposed as the source of neutrinos at a neutrino factory $[1,2]$ and as the means to deliver multi-TeV lepton-antilepton collisions at a muon collider [3,4]. In such facilities where the muon beam is produced from the decay of pions generated by a high-power proton beam striking a target, the muon beam occupies a large volume in phase space. To optimise the muon 
yield while maintaining a suitably small aperture in the muon-acceleration system requires that the muon beam be 'cooled' (i.e., its phase-space volume reduced) prior to acceleration. Recently, a muon-collider scheme based on the production of $\mu^{+} \mu^{-}$pairs through the annihilation of positrons impinging on a target has been proposed [5]. These $\mu^{+} \mu^{-}$pairs are created close to threshold which restricts the phase-space volume occupied by the muons and which could therefore produce beams with small emittance at energies $\gtrsim 20 \mathrm{GeV}$.

A muon is short-lived, decaying with a lifetime of $2.2 \mu \mathrm{s}$ in its rest frame. Therefore, beam manipulation at low energy $(\leq 1 \mathrm{GeV})$ must be carried out rapidly. Four cooling techniques are in use at particle accelerators: synchrotron-radiation cooling [6]; laser cooling [7-9]; stochastic cooling [10]; and electron cooling [11]. In each case, the time taken to cool the beam is long compared to the muon lifetime. In contrast, the cooling time associated with ionization cooling, in which the energy of a muon beam is reduced as it passes through a material - the absorber - and is subsequently accelerated, is short enough to allow the muon beam to be cooled efficiently with acceptable decay losses. Ionization cooling is therefore the technique by which it is proposed to reduce the muon-beam phase space in a proton-derived neutrino factory or muon collider [12-16]. This technique has never been demonstrated experimentally and such a demonstration is essential for the development of future high-brightness muon accelerators.

The international Muon Ionization Cooling Experiment (MICE) was designed [17, 18] to perform a full demonstration of transverse ionization cooling. Particle densities in the ionizationcooling channels that are conceived for the neutrino factory or muon collider are low enough for collective effects such as space charge to be neglected. This allowed the MICE experiment to record muon trajectories one particle at a time. The beam-cooling effect was produced by placing an energy-absorbing material in the bore of a superconducting solenoid that was used to focus and transport the muon beam. The MICE collaboration has taken the data necessary to study the beam-cooling properties of lithium-hydride and liquid hydrogen. This paper describes the design and construction of the containment vessel for the liquid hydrogen and the associated cryogenic and gas systems, together with a summary of the safety engineering and the control and monitoring system. The performance of the system in cool-down, hydrogen liquefaction, and stable operation is also presented.

\section{The Muon Ionization Cooling Experiment}

\subsection{Beamline}

The muons for MICE were created from the decay of pions produced when a target dipped into the circulating proton beam in the ISIS synchrotron at the STFC Rutherford Appleton Laboratory (RAL) [19, 20]. A beamline of nine quadrupoles, two dipoles, and a superconducting 'decay solenoid' collected each burst of particles (a 'spill') and transported the momentum-selected particles to the experiment [21]. The small fraction of pions that remained in the beam were identified, and subsequently rejected during analysis, using the time-of-flight hodoscopes and Cherenkov counters that were installed in the beamline upstream of the experiment [22].

The experiment contained an absorber/focus-coil (AFC) module sandwiched between two spectrometer modules, as shown in figure 1. The focus-coil (FC) module had two separate windings 


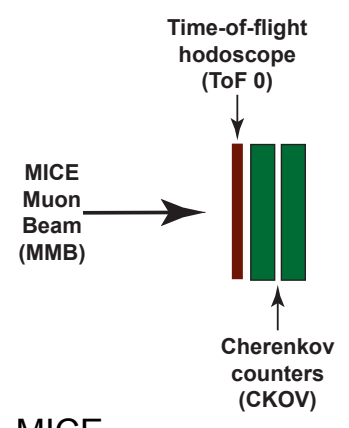

MICE

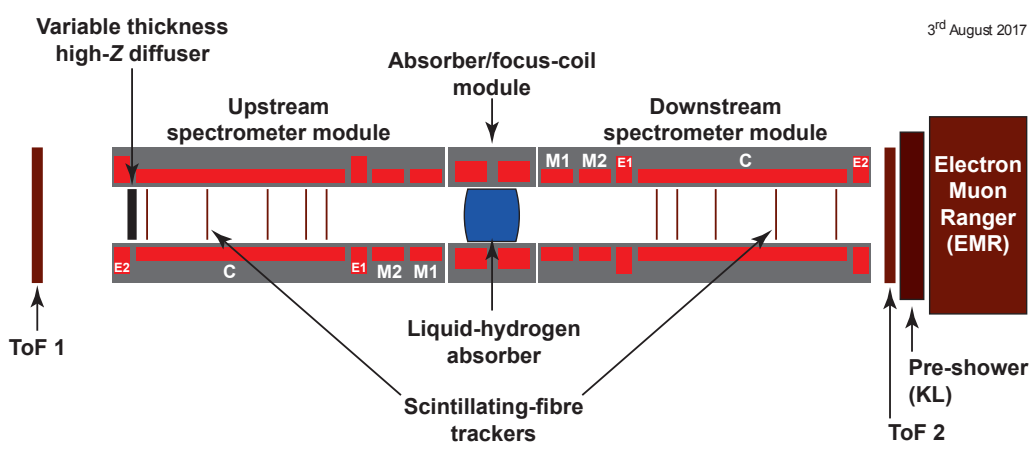

Figure 1. Schematic of the experiment. The red rectangles represent the superconducting coils. The individual coils in the spectrometer modules are labelled thus: E2, C, E1, M2, M1 in the upstream module, and M1, M2, E1, C, E2 in the downstream module. The various detectors (time-of-flight hodoscopes [23, 24], Cerenkov counters [25], scintillating-fibre trackers [26], KLOE Light (KL) calorimeter [21, 27], and electron muon ranger [28]) used to characterise the beam are also represented.

to produce either aligned or opposed magnetic fields. An absorber, such as lithium hydride $(\mathrm{LiH})$ or liquid hydrogen $\left(\mathrm{LH}_{2}\right)$, was placed at the centre of the FC module to 'cool' the beam.

Each spectrometer module contained a long centre coil (C) with two end-correction coils (E1, E2), and two 'match' coils (M1, M2). The emittance was measured upstream and downstream of the cooling cell using scintillating-fibre tracking detectors [26] immersed in the uniform magnetic field provided by the coils (E1, C, E2). The trackers were used to measure the trajectories of individual muons before and after they had traversed the absorber. These reconstructed trajectories were combined with information from instrumentation upstream and downstream of the spectrometer modules to measure the muon-beam emittance at the upstream and downstream tracker reference planes [29]. A diffuser was installed at the inlet of the upstream spectrometer module to vary the initial emittance of the beam [30]. The instrumentation upstream and downstream of the spectrometer modules enabled the selection — for analysis — of a pure sample of muons. The 'match' coils were used to match the beam optics between the uniform-field region and the neighbouring FC.

\subsection{Safety considerations}

The MICE hydrogen system was designed to store $22 l$ of liquid hydrogen in an aluminium vessel at $\sim 20 \mathrm{~K}$ and slightly above atmospheric pressure. Hydrogen/air mixtures are explosive between the lower explosive limit (LEL) of $4 \%$ and the upper explosive limit (UEL) of $77 \%$ hydrogen by volume [31]. Therefore, it was essential to prevent the ingress of air into the hydrogen system and to prevent the escape of hydrogen into the experimental hall. Any venting of gas that may have been contaminated with hydrogen had to be controlled such that it was purged into the atmosphere at a concentration well below the LEL.

The philosophy adopted was to design a primary system to contain the hydrogen over a range of temperatures and pressures that would exceed the range expected during operation. The room-temperature parts of this primary system were enclosed within a secondary containment system which was continually flushed with dry nitrogen gas. This ensured that any leak from this part of the primary system was contained within an inert medium and continually flushed out to 
atmosphere. The cooled part of the primary system was surrounded by an insulating vacuum which was continuously pumped and the exhaust released to atmosphere. The entire hydrogen system, i.e. primary and secondary containment, was engineered to be robust to two unrelated failures, whilst maintaining safety in operation. To meet these requirements a number of features were designed into the system:

- Gas lines from bottle to point of use: constructed from double-walled stainless-steel piping with continuous flow of dry nitrogen gas through the jacket. The internal tube and the jacket were both leak tested, and leak rates below $10^{-6} \mathrm{mbar} l / \mathrm{s}$ were required;

- Process and control valves: housed within a cabinet which was maintained at a pressure slightly below local ambient. The cabinet was continually purged with dry nitrogen gas and vented through the roof;

- Low-temperature circuit: designed to ensure that there was no possibility of isolation of any volume of hydrogen due to freezing, thereby preventing any possible over-pressure and vessel-failure scenarios; and

- Low-temperature circuit insulation vacuum: its pumping system was vented via ATEX-rated pumps external to the experimental area and within a locked cabin to prevent personnel access during operation. The vacuum-volume leak rate was verified to be below $10^{-8} \mathrm{mbar} l / \mathrm{s}$.

All leak rates were measured using a helium leak detector when the system was warm and, where relevant, verified once cold. Any air leaking into the vacuum of the secondary system would have plated out on the cold surfaces and then been released if the system had warmed significantly for any reason. Such a warm-up would be expected if a significant leak of hydrogen into the insulating vacuum had occurred, potentially producing an explosive mixture of hydrogen and air. The maximum leak-rate into the vacuum was therefore chosen to restrict the volume of plated gas accumulated over the operating period of the experiment to a safe value. Leak rates below $10^{-8} \mathrm{mbar} l / \mathrm{s}$ would have resulted in a maximum cryo-pumped air volume of $3 \times 10^{-4}$ bar $l$ per year, which was considered sufficiently low.

\subsection{Safety approval process}

The Science and Technology Facilities Council (STFC) carries a legal obligation to comply with the requirements of the Health and Safety at Work Act, 1974. This responsibility is articulated by STFC's Safety, Health and Environment (SHE) group in the form of safety codes, several of which governed the implementation of the MICE liquid-hydrogen system. These codes map onto external frameworks such as the Pressurised Equipment Directive (PED) and the Dangerous Substances and Explosive Atmospheres Regulations (DSEAR). To ensure compliance with these requirements, the MICE liquid-hydrogen project underwent a number of safety reviews at crucial points during design and construction. In addition to pre-operation reviews carried out by STFC staff with external experts in October 2011 and January 2015, the reviews listed below were conducted by independent external consultants: 
- June 2006. Preliminary HAZOP review of the design of the liquid-hydrogen system as a single entity. Recommendations were made for the control systems, redundancy, interlocks, and protection against failure of major components [32].

- November 2010. A Safety Integrity Level (SIL) rating and a Layers of Protection Analysis (LOPA) were carried out on the latest design [33]. An internal review committee then gave approval for hydrogen R\&D work to begin in October 2011, but also requested a second HAZOP which would focus on procedures.

- October 2012. Second HAZOP review that focused mainly on the operational aspects of the system within the overall experimental context to account for influences on the hydrogen system from nearby equipment, and also to validate design and hardware changes since previous exercises [34].

- May 2017. A final HAZOP was requested by STFC to consider changes that had been made to increase the capacity of the system's pressure relief arrangements, other more-minor changes to the design, and operational factors [35]. The successful conclusion of this HAZOP led to the final approval by STFC for fully running the liquid hydrogen system in MICE.

Some of the key pieces of equipment, such as the absorber vessel and the aluminium windows, were manufactured by international collaborating institutes and therefore could not directly comply with PD5500, the UK standard for pressure vessel approval. In these cases, an EU Declaration of Conformity was compiled that connected the design and manufacturing data with local pressure system frameworks. These documents were then authorised at Director level within the manufacturing institutes. This procedure, when combined with pressure tests in the UK, then enabled approval and registration within the UK.

All persons involved in the hardware and operations of the liquid-hydrogen system were obligated to obtain and maintain their training on working safely with explosive atmospheres by attending courses, such as this example [31].

\section{Absorber vessel}

Drawings of the AFC module and the installed absorber vessel are shown in figure 2. The absorber vessel was set at the centre of the FC magnet coils. The diameter of the warm bore was $470 \mathrm{~mm}$ and its length was $844 \mathrm{~mm}$. The diameter of the flange by which the FC was connected mechanically to the spectrometer module was $1,514 \mathrm{~mm}$.

\subsection{Design considerations}

As a muon beam passes through material, some of the kinetic energy of the muons is lost through ionization of the material. This process results in a reduction of the normalised transverse emittance and the beam is said to be cooled. Muons will also undergo multiple Coulomb scattering which increases the divergence of the beam, thereby increasing the normalised transverse emittance and heating the beam.

Ionization-energy loss is characterised by $\frac{d E}{d x}$, where $E$ is the muon energy and $x$ is the distance travelled within the absorber. Multiple Coulomb scattering is characterised by the radiation length, 

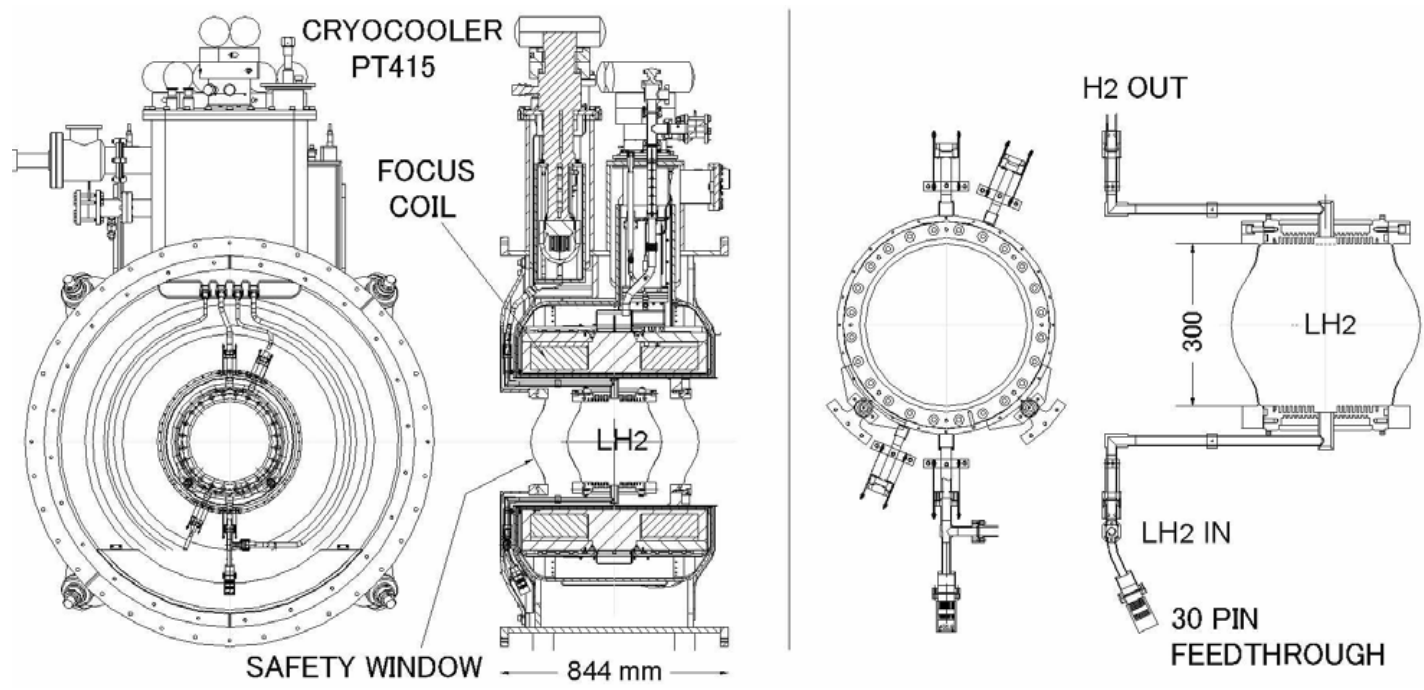

Figure 2. Left panel: Drawing of the absorber/focus-coil (AFC) module showing the principal components. Right panel: detail of the liquid-hydrogen absorber vessel.

$X_{0}$. For liquid hydrogen, $\frac{d E}{d x} \sim 0.03 \mathrm{MeV} / \mathrm{mm}$ and $X_{0} \sim 8905 \mathrm{~mm}$ [36]. The absorber vessel was manufactured using aluminium for which $\frac{d E}{d x} \sim 0.4 \mathrm{MeV} / \mathrm{mm}$ and $X_{0} \sim 90 \mathrm{~mm}$ [36]. To maximise the cooling effect from energy loss in liquid hydrogen, while minimising the heating effect from multiple Coulomb scattering in the aluminium windows, these windows were required to be as thin as possible [37]. Safety considerations, as described in section 2.2, required a secondary containment system. Therefore, the absorber vessel was situated in an evacuated space within two more thin aluminium safety windows, so the muon beam had to traverse four windows, as shown in the left panel in figure 2 .

\subsection{Absorber vessel body}

The absorber vessel comprised a cylindrical aluminium body sealed with two thin aluminium end windows, as shown in the right panel of figure 2. The absorber vessel was specified to contain $22 l$ of liquid, so the body had an inner diameter of $300 \mathrm{~mm}$ and a length between its end flanges of $230 \mathrm{~mm}$. The length along the central axis between the two domes of the thin aluminium end windows was $\sim 350 \mathrm{~mm}$. The body contained an annular cooling channel within its walls that could act as a heat exchanger. This channel was designed to allow the possibility of cooling the vessel body externally [38]. However, it was found that this cooling was not necessary in MICE because the absorber vessel cooled sufficiently quickly with cold gas from the condenser, as described in section 7.1. Small indium-sealed flanges connected the aluminium pipes from the absorber vessel to the stainless-steel pipes from the condenser.

Figure 3 shows a photograph of the inside of the absorber vessel body. The two flanged windows were sealed to the end flanges of this body using indium contained in grooves. The heat exchanger fins [38] and five pairs of thermometers (LakeShore Cernox 1050-SD) are visible in this photograph. These five thermometer pairs were inside the vessel at locations spaced by $45^{\circ}$ around the circumference and were monitored with a LakeShore 218S. Each pair monitored 


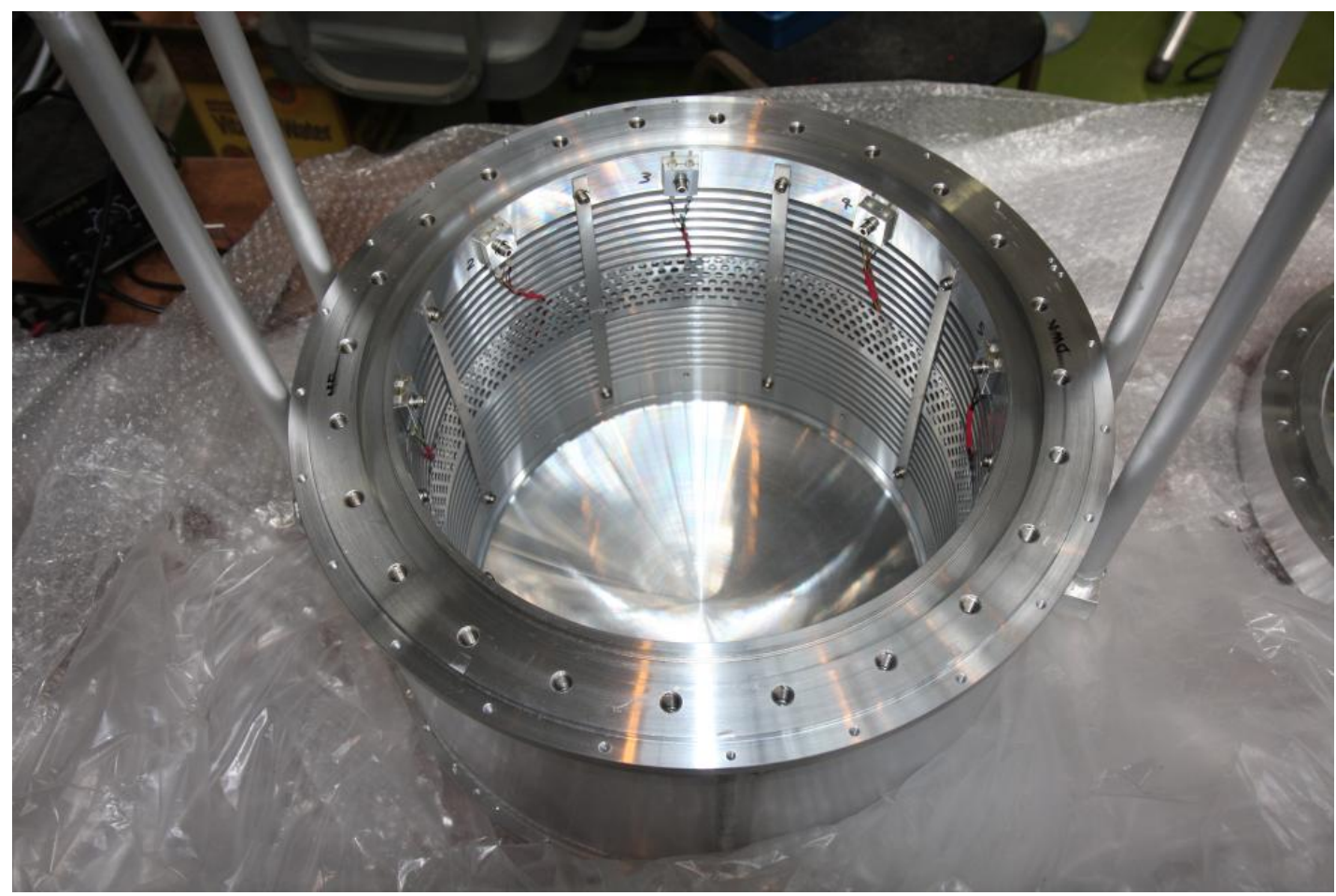

Figure 3. Photograph of the absorber vessel body.

the presence of liquid hydrogen at that position; one of these Cernox sensors was operated with a small current as a thermometer, and the other was occasionally heated by a pulse of larger current. The difference between the two measured temperatures was small when these sensors were in liquid due to good cooling efficiency, but the difference was larger when these sensors were in gas since heat transport through the gas is worse than in the liquid. The sensor wires were extracted to vacuum part-way along the liquid-hydrogen inlet pipe at a 30-pin hermetic feed-through, as shown in figure 2. Signals from each sensor were carried on two wires inside the absorber vessel, between the sensor and the feed-through, and by four wires in the vacuum outside the vessel. Two Cernox thermometers and two heaters (LakeShore HR-25-100) were mounted externally on each end flange. Two additional Cernox thermometers were mounted externally on the hydrogen inlet and outlet lines. These thermometers were exposed to vacuum and thermal radiation so the thermometry here was less accurate than that inside the absorber vessel, but gave indications of the flow of cooling gas in the circuit. To minimise heat input from contact with the magnet bore, the absorber vessel was mounted on glass-epoxy (G10) supports of low thermal conductivity. To minimise radiative heat input, multilayer insulation (MLI) was wrapped around the absorber vessel and all the low-temperature pipework. The number of layers of MLI over the end windows was first entered into a Monte Carlo program to check that the scattering of muons by the MLI was insignificant compared to that of the windows, before the vessel was integrated into the system and cooled. 

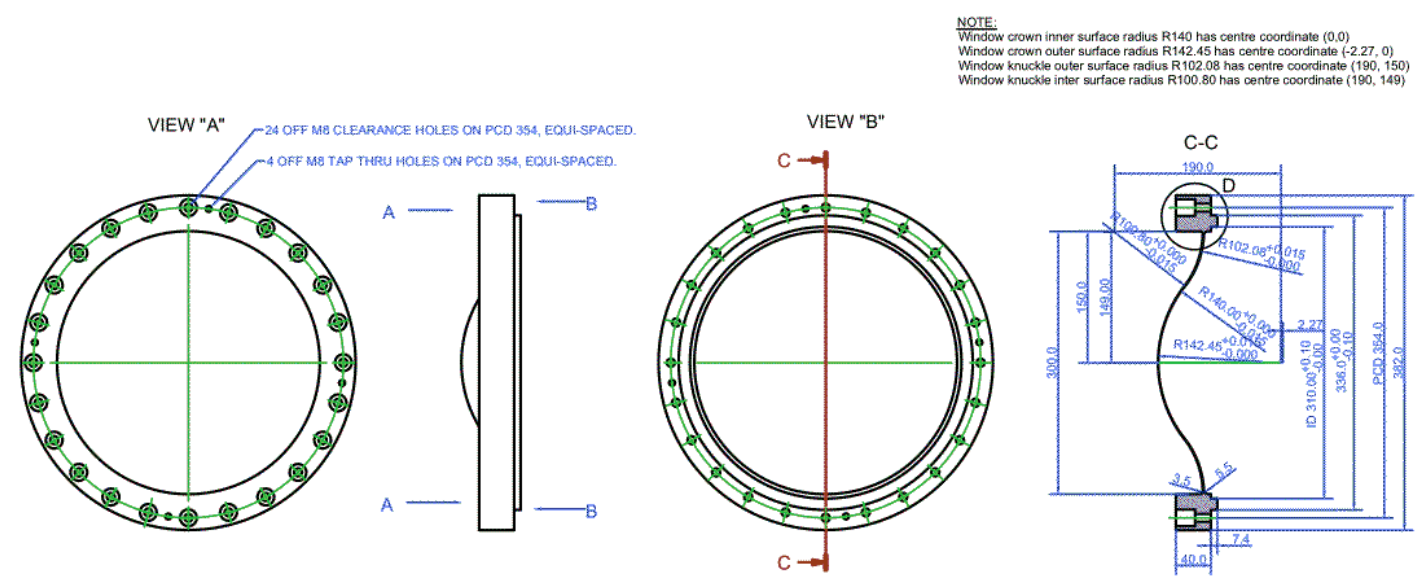

Figure 4. Aluminium absorber vessel window, designed to have a central thickness of $180 \mu \mathrm{m}$, for the containment of liquid hydrogen. Both types of safety windows were similar to the vessel window, but had a central design thickness of $210 \mu \mathrm{m}$.

\subsection{Windows}

The liquid hydrogen was contained between two aluminium windows, each having a thickness of $\sim 180 \mu \mathrm{m}$ at the centre and increasing to $\sim 360 \mu \mathrm{m}$ near the outer flange. Aluminium safety windows, each with a central thickness of $\sim 210 \mu \mathrm{m}$, enclosed the absorber vessel in the magnet bore. Thin aluminium was chosen to minimise multiple scattering. Thinner windows lead to less scattering and more muon-beam cooling. Although a MICE window with a central thickness of $125 \mu \mathrm{m}$ had successfully been machined using alloy 6061-T651, it would not withstand enough pressure. The pressure in the absorber vessel reached 1500 mbar during typical operations. The aluminium alloy we chose to use (6061-T651) was assayed to contain $0.61 \%$ silicon, $0.26 \%$ iron, $0.25 \%$ copper, $0.02 \%$ manganese, $1.02 \%$ magnesium, $0.20 \%$ chromium, $0.01 \%$ zinc, $0.05 \%$ titanium, $0.01 \%$ zirconium, $0.15 \%$ maximum other material, and at least $97.42 \%$ aluminium (all measured by weight). The yield strength was measured at room temperature to be $275 \pm 5 \mathrm{MPa}$, although this would be greater at $20 \mathrm{~K}$. A drawing of a MICE absorber vessel window is shown in figure 4 . The double-bend geometry increases the burst strength [37,39].

\subsubsection{Window manufacture}

A CNC Fadal 5020A vertical machining centre and a CNC Romi lathe with a 27 inch swing were used to machine the windows from a solid block of aluminium alloy. Precision backing plates supported the windows during this process. Each window was machined to a $2000 \mu \mathrm{m}$ central thickness, and then measured with the micrometer jig shown in figure 7. The window was then returned to the lathe for final machining while the lathe still had the positions stored in its memory. Clear plastic cases were fabricated to protect the windows from damage in transit, while still allowing visual inspection. Finished windows can be seen in figures 5 and 8. 
Table 1. Results of measuring the central thickness of the three types of windows with the View Precis 3000 Optical CMM shown in figure 5. The windows actually used in MICE were numbers 002, 003, 009, and 014 .

\begin{tabular}{|ccccc|}
\hline $\begin{array}{c}\text { Window } \\
\#\end{array}$ & $\begin{array}{c}\text { Window } \\
\text { Type }\end{array}$ & $\begin{array}{c}\text { Central Thickness } \\
\text { Measured }(\mu \mathrm{m})\end{array}$ & $\begin{array}{c}\text { Central Thickness } \\
\text { Design }(\mu \mathrm{m})\end{array}$ & Note \\
\hline 001 & Absorber & & 180 & \\
002 & Absorber & $174 \pm 5$ & 180 & \\
003 & Absorber & $184 \pm 2$ & 180 & \\
004 & Absorber & & 180 & \\
005 & Absorber & $176 \pm 6$ & 180 & flaw at centre \\
006 & Safety I & $222 \pm 6$ & 210 & \\
007 & Safety I & & 210 & \\
008 & Safety II & $233 \pm 5$ & 210 & \\
009 & Safety II & $230 \pm 9$ & 210 & \\
010 & Absorber & & 180 & \\
011 & Absorber & & 180 & \\
012 & Safety I & $197 \pm 7$ & 210 & \\
013 & Safety I & & 210 & \\
014 & Safety I & $197 \pm 8$ & 210 & \\
\hline
\end{tabular}

\subsubsection{Window thickness measurement}

The thicknesses of three different types of finished windows (one absorber and two safety) were measured with the View Precis 3000 Optical Co-ordinates Measurement Machine (CMM) shown in figure 5. The complete surface profile of a window was measured with the laser on one side, and then the window was turned over to measure the other side. The difference between the surface profiles of both sides of the window gave the thickness. Three tuning balls were glued to the window to establish the reference coordinate system; key to getting a good measurement was to establish the same reference coordinate system for both sides of each window. Some results of the measurements are shown in table 1 and figure 6. For some of the windows, the thickness measurement was checked by scanning only the small area around the window centre with a very dense meshing. This gave a more accurate measurement of the thickness at the window centre.

Before adopting the CMM method just described, radioactive sources were used to measure the thickness of a prototype window machined to the MICE absorber window profile. This was done before individual numbers were assigned to each window that would be a candidate for use in the experiment. The source and detector (Geiger tube) were on opposite sides of the window so there was no need to move the window during this process, as would be required in the CMM method. Low energy electrons are strongly attenuated by modest thicknesses of aluminium. This validation test was optimised to measure the central window thickness by choosing two different beta sources, ${ }^{90} \mathrm{Sr}$ and ${ }^{204} \mathrm{Tl}$, with electron energies that have a half-range of about $180 \mu \mathrm{m}$ in aluminium. The 


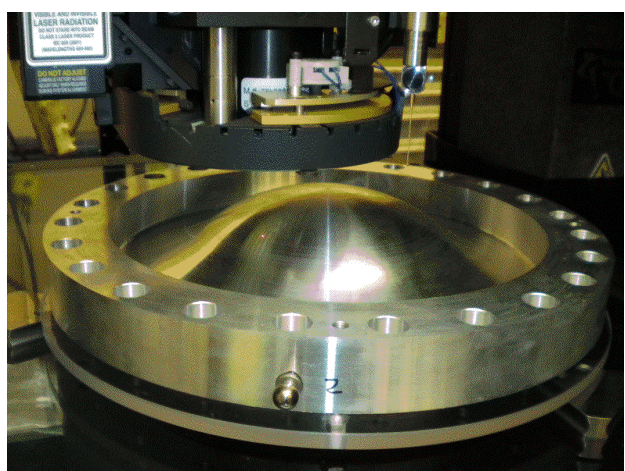

Figure 5. The View Precis 3000 Optical CMM measured the surface profile of each window, one side at a time.

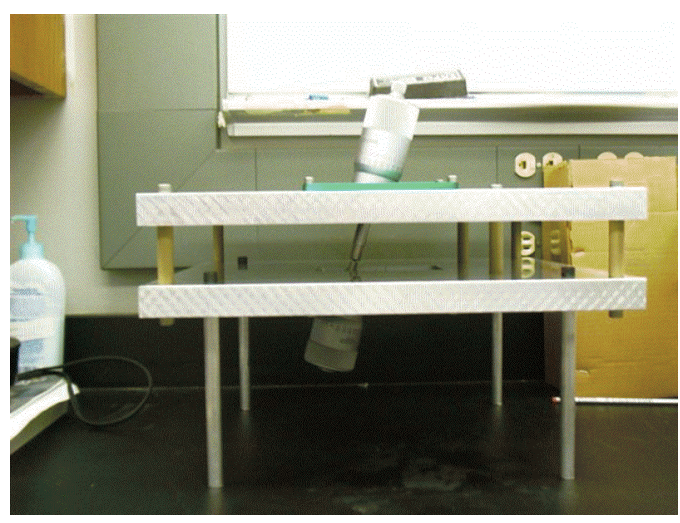

Figure 7. Jig for measuring window thickness at the centre and at $15^{\circ}$ from the peak of the dome with a pair of Starrett T465 micrometers accurate to 3 microns.

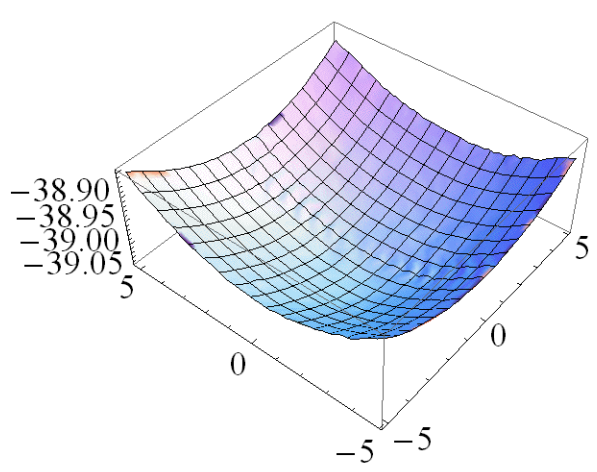

Figure 6. Result of the CMM measurement of one side of one window. All the axes are labelled in units of millimetres.

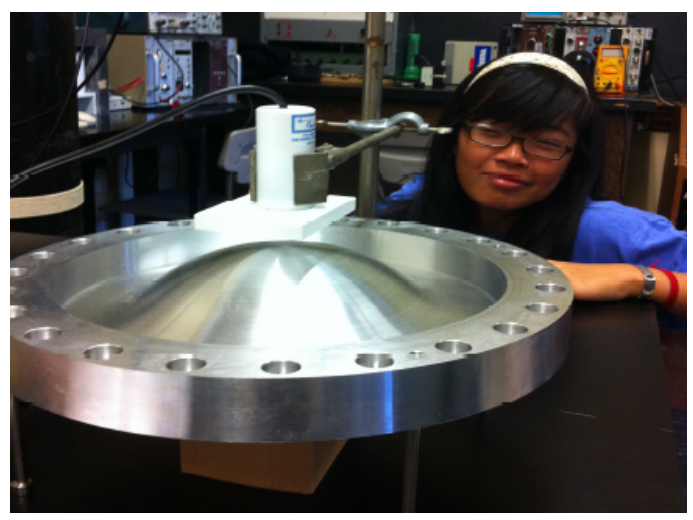

Figure 8. $\quad{ }^{90} \mathrm{Sr}$ and ${ }^{204} \mathrm{Tl}$ beta sources and a Geiger tube were used to check the central thicknesses of windows.

attenuation of electrons in a thin sheet of material of thickness $x$ was described using the equation:

$$
R=A e^{\alpha x}+B e^{\beta x}+C .
$$

Due to electron scattering, the result can be sensitive to apparatus geometry so a careful calibration was performed using aluminium sheets of known thickness with counts being accumulated for 10 minutes per sheet. The central thickness of the absorber window in figure 8 was measured to be $178 \pm 6$ (stat) \pm 4 (fit) $\mu \mathrm{m}$. This result showed that the machining procedure was sufficiently accurate and that the preferred method of measurement (CMM) used for later windows had no significant systematic error.

\subsubsection{Window burst tests}

The windows were designed to withstand internal pressures from vacuum ( -1.0 bar $)$ to a minimum of 96 psig. (6.6 bar). Two absorber windows (figure 9) and one safety window (figure 10) had been destructively burst-tested at room temperature. A dial indicator showed the deflection up to the 


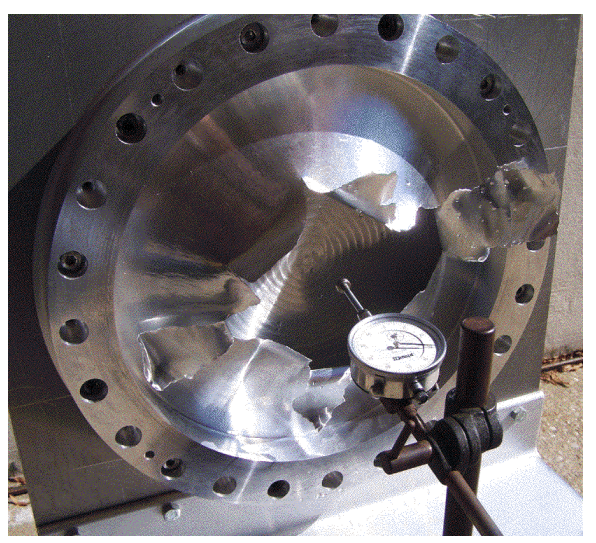

Figure 9. This absorber vessel window burst when pressurised with water.

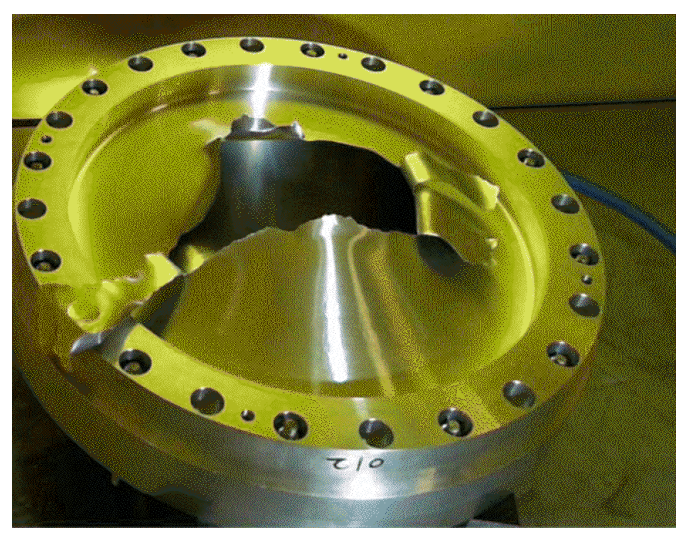

Figure 10. This Type I safety window (number 012 in Table 1) burst when slowly pressurised with nitrogen gas.

moment of burst. The absorber windows burst at 8.27 bar and 8.41 bar, respectively, and the Type I safety window burst at 7.67 bar.

\section{Condensing unit}

The condenser was situated above the absorber vessel and both were inside the thermally-insulating vacuum of the safety volume inside the cryostat, as shown in figure 11 . The condenser was cooled through direct contact with the second stage of a coldhead in order to cool, and ultimately liquefy, the incoming gas to fill the absorber vessel with liquid. The condenser was suspended from the underside of the top-plate of the cryostat by four stainless-steel rods, with couplings that allowed for differential contraction of the rods and the coldhead. These rods were heat-sunk to the thermal shield to reduce the thermal conduction to the condenser along these four rods to $\sim 0.15 \mathrm{~W}$. Inside the condenser was an array of copper fins to facilitate the removal of heat from the gas. These fins were part of a large copper block, fitted into the curved side of the stainless-steel condenser, to which the second stage of the coldhead was firmly connected, as can be seen in figure 12 .

The condenser was cooled by the second stage of the two-stage coldhead (Cryomech PT415 pulse tube); this second stage has a nominal cooling capacity of $1.5 \mathrm{~W}$ at $4.2 \mathrm{~K}$ and base temperature of $2.8 \mathrm{~K}$. The first stage of the coldhead cooled the thermal shield that surrounded the condenser to about $45 \mathrm{~K}$, as well as the incoming gas via thermal links between this shield and the gas-filled tubes, as shown in figure 13. The thermal shield was covered with three blankets of multi-layer insulation (MLI); each blanket comprised ten layers of Mylar coated with reflective aluminium film, the layers separated by polyester netting. The $15 \mathrm{~mm}$ bore stainless steel pipework between the condenser and the absorber vessel was covered with two blankets of MLI. The absorber vessel, including its thin windows, was covered with four blankets of MLI. For lack of space, it was not possible to install a cooled thermal shield around the pipework and the absorber vessel; this would have improved the rates of cooling and liquefaction. The weight of the pipework near the bottom of the absorber vessel was suspended by six strands of $0.5 \mathrm{~mm}$ diameter nickel alloy wire of length 


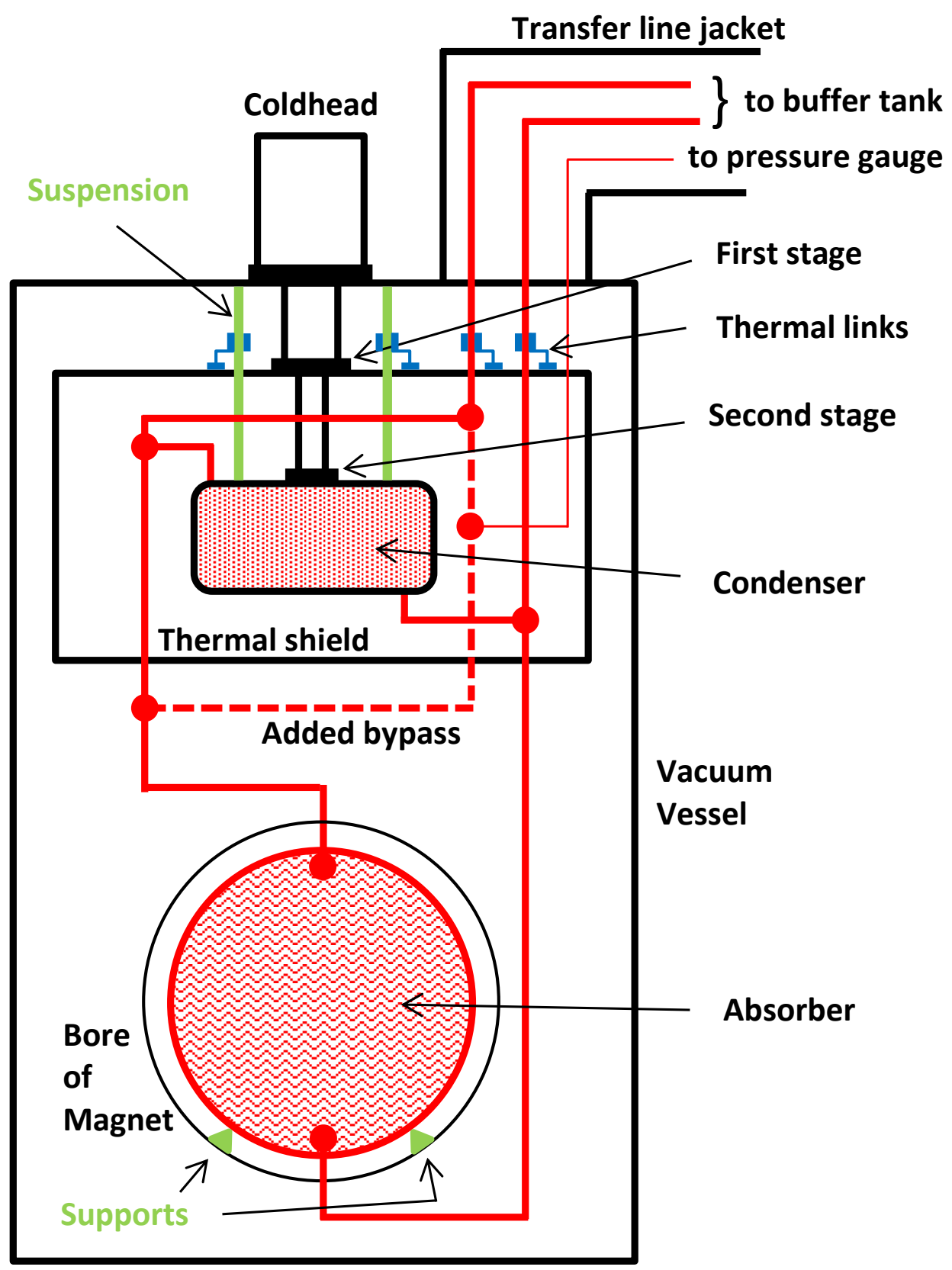

Figure 11. Schematic of the condensing system. The bypass loop added as an extra precaution against blockage by solidified hydrogen is shown as a dashed line. 


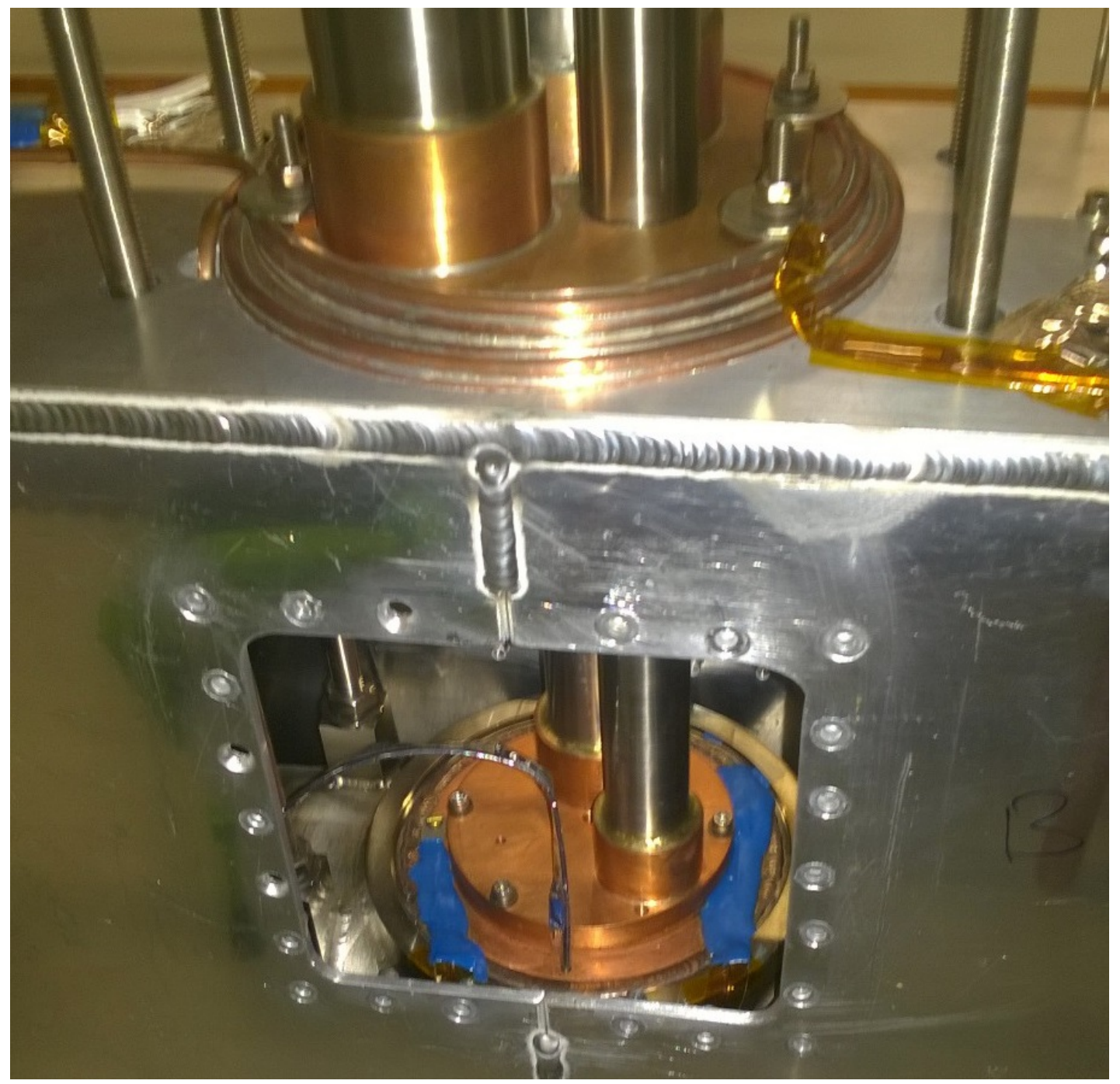

Figure 12. Through the access window in the thermal shield can be seen the bolted thermal connection between the second stage of the coldhead and the condenser. The condenser is mostly hidden inside the welded aluminium thermal shield that was connected (at the top of the picture) to the first stage of the coldhead. The window was blanked with an aluminium plate before the shield was fully enclosed in blankets of super-insulation for the cooldown. The blue stycast bonded the wires of the two thermometers to the copper plate for good heat-sinking. At the front of the copper plate, the vertical wire bundle wrapped in super-insulation connected to the heater which had been inserted into a drilled hole. This control heater prevented the condenser from getting too cold and thus prevented freezing of the hydrogen. 


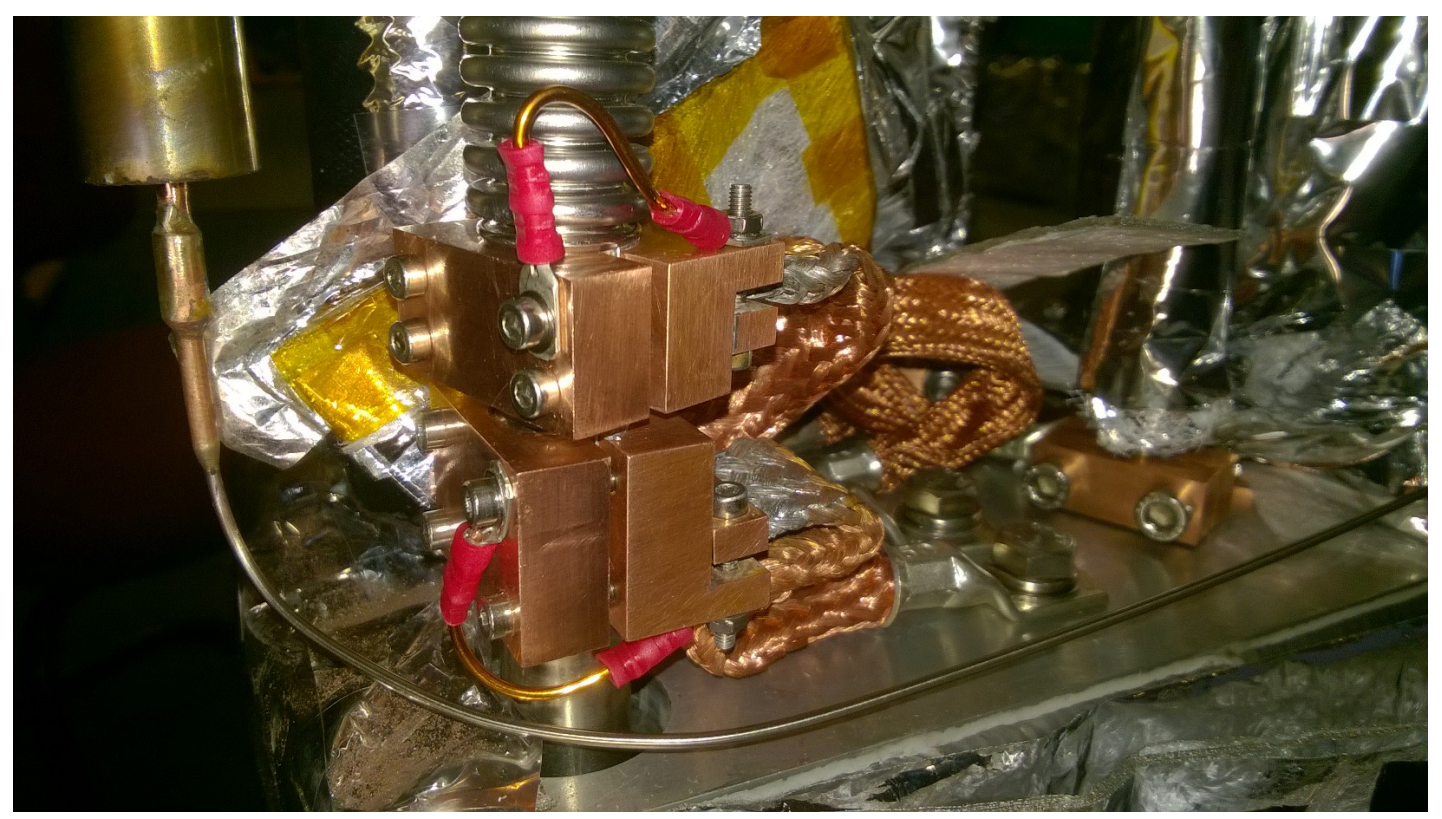

Figure 13. Photograph showing some of the copper heat-links between the gas-filled tubes and the thermal shield before being covered with three blankets of MLI.

$450 \mathrm{~mm}$. These low-thermal-conductivity wires made a negligible contribution of about $0.01 \mathrm{~W}$ to the heat-load.

To avoid the possibility of blocking the pipework due to the formation of hydrogen-ice (under fault conditions which could cause temperatures lower than $\sim 14 \mathrm{~K}$ in the condenser), a bypass loop was included as shown in figures 11 and 14. The lower end of the bypass was at the (never-freezing) boil-off side of the absorber pipework and the upper end was at the (never-freezing) first stage of the coldhead, the temperature of which could never go below $\sim 35 \mathrm{~K}$. Thus, the bypass line always provided an ice-free path from the absorber vessel to the pressure relief valves. This bypass line had an insignificant effect on the cooling efficiency.

\section{Gas system}

\subsection{Overview of gas flows}

A schematic of the flows in the hydrogen gas system is shown in figure 15. The system comprised a hydrogen-gas panel inside an enclosure, a source of hydrogen gas, the condensing unit, a pump for evacuating hydrogen from the pipework, a pump-set to evacuate the safety vacuum around the condensing unit, and nitrogen-gas flushing of the secondary containment. This system (from the source, through the gas panel, to the connections to the absorber vessel) was fabricated entirely from stainless-steel components. Pipework was welded wherever possible, X-rayed to ATEX rating where required, and non-welded joints were sealed using metal gaskets. The pipework was thoroughly tested for leaks using helium gas.

Hydrogen gas was obtained from a multi-cylinder pack (MCP) situated in a locked cage outside the experimental hall. Also inside this cage were the regulator and the pressure-relief valves for 


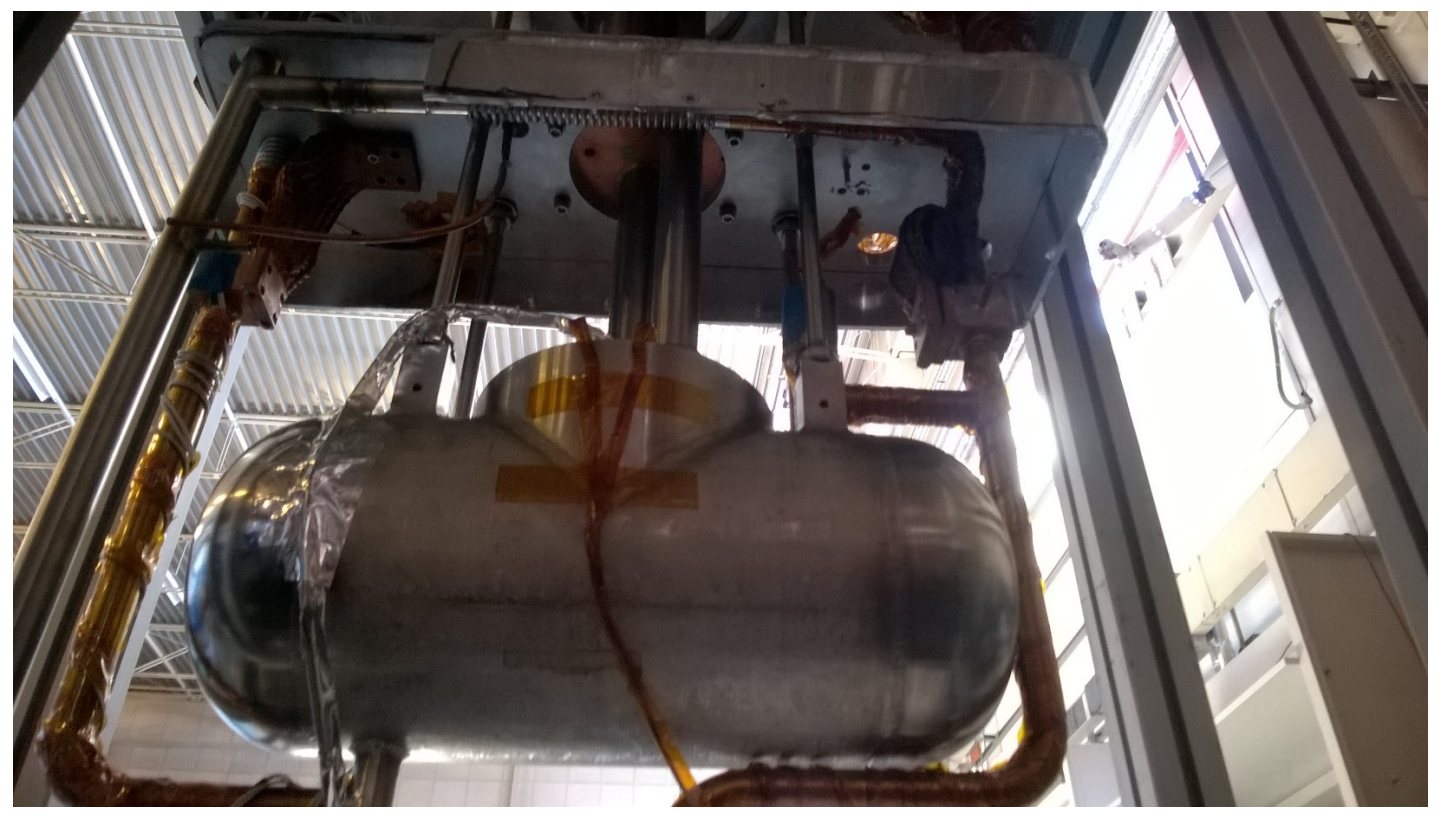

Figure 14. Photograph showing the condenser with the bypass loop added. The aluminium thermal shield had been cut away for this modification and was re-welded for the following runs.

the section of pipework outside the building. This MCP contained ample gas, thus obviating the need to break and remake connections which would have risked contamination with air. It initially contained 200 bar and, by the end of the experiment, 170 bar remained, corresponding to a total consumption of roughly 20,000 bar $l$ of gas.

The hydrogen gas flowed from the regulator in the cage to the gas panel inside the experimental hall via a jacketed line which was formed from two concentric tubes. The inner tube carried the hydrogen gas while the outer tube carried nitrogen gas flowing at a rate of $\sim 1 / / \mathrm{min}$. to flush out any hydrogen that might leak from the inner tube. This nitrogen gas flowed into the enclosure that surrounds the gas panel and was then extracted, via large diameter ( $>250 \mathrm{~mm}$ bore) pipes, by fans on the roof of the building and expelled to the atmosphere approximately $4.5 \mathrm{~m}$ above roof level.

The condensing unit was supplied with room-temperature gas via a transfer line comprising: two $22 \mathrm{~mm}$ bore tubes to convey the gas (initially designed as a separate feed and return but later used in parallel); one $\sim 4 \mathrm{~mm}$ bore tube which enabled a pressure gauge (PG-04) situated in the gas-panel enclosure to measure the gas pressure above the absorber vessel; and a surrounding pipe (110 $\mathrm{mm}$ bore) which formed the jacket for the flowing nitrogen gas $(\sim 1 / / \mathrm{min}$.) that would carry any leaked hydrogen gas back into the gas-panel enclosure from where it would be safely extracted. The pressure of the gas supplied to the condensing unit was measured at the outlet of the gas panel (PG-02). Any significant difference in pressure between PG-02 and PG-04 would have revealed a blockage in the pipework at low temperatures.

There were ten hydrogen sensors, in pairs at five positions:

- In the enclosure to detect leakage from the hydrogen-filled pipework to the nitrogen gas jacket; 


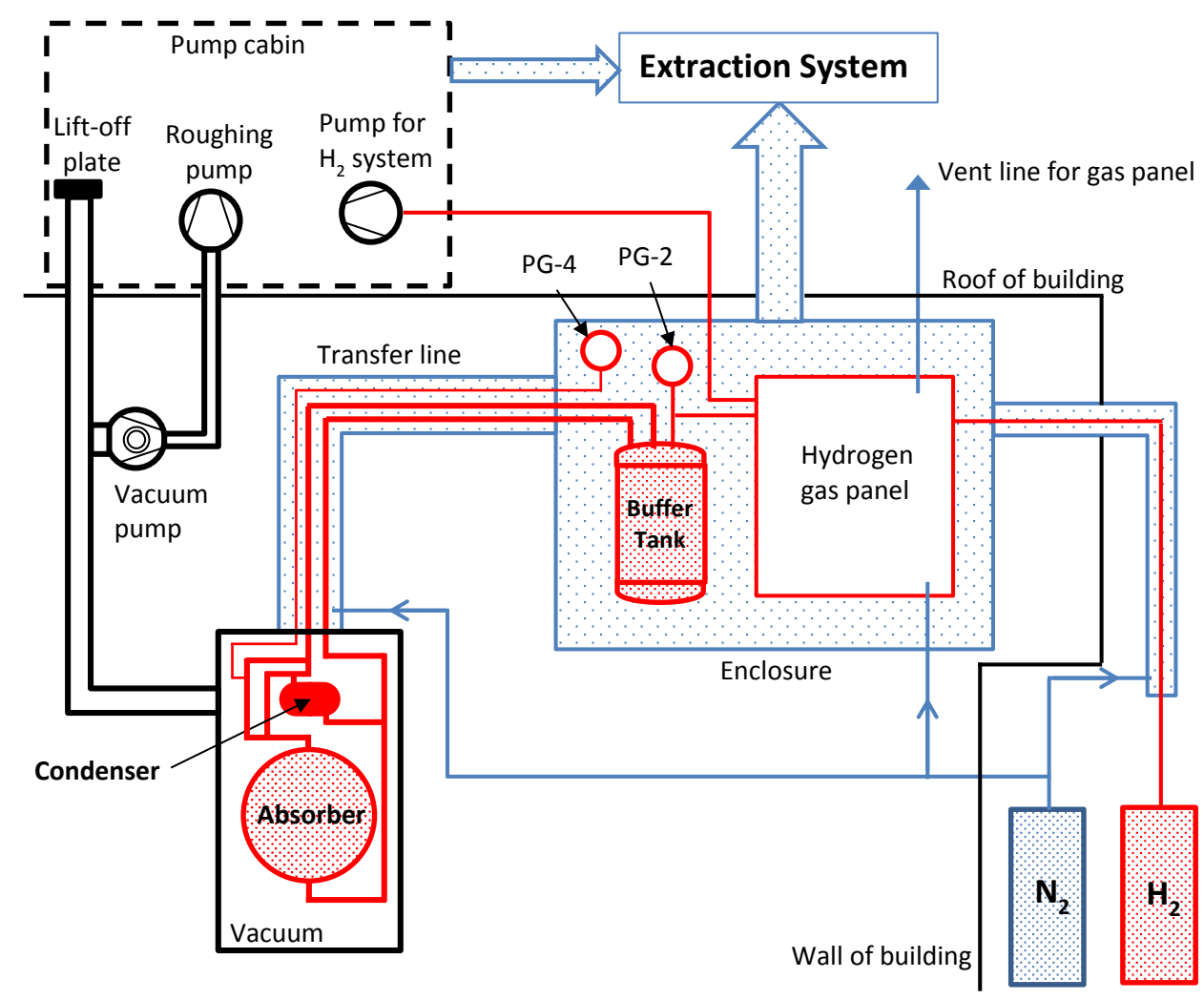

Figure 15. Gas flows in the hydrogen gas system: hydrogen gas is shown in red, nitrogen gas is shown in blue. The helium gas used for initial flushing of the hydrogen volume, and during cooling, is not shown.

- In the extraction system for the same reason;

- In the pump cabin to detect leakage into the safety vacuum around the condensing unit or hydrogen pumped from the gas panel;

- In the vent line that is purged continuously by nitrogen gas; and

- In the experimental hall to detect hydrogen gas leakage from the secondary containment system.

\subsection{Safety volume and quench line}

The absorber vessel was situated in the bore of the focus-coil magnet, with thin aluminium safety windows on both sides forming a safety volume (SV), as shown in figure 2 . The safety windows were thin to minimise scattering of the muon beam. Therefore, safe pump-down and venting procedures were followed to ensure that pressure differentials across all safety and vessel windows were not large enough to compromise their function. Three Leybold Ceravac CTR 100 transducers of overlapping ranges measured the vacuum pressure in the SV between $\sim 10^{-4}$ mbar and 1000 mbar. The SV was evacuated to $\sim 10^{-4}$ mbar before cooling began, to provide a thermally-insulating environment for the condensing unit, and was then pumped continuously to extract any hydrogen that may have 


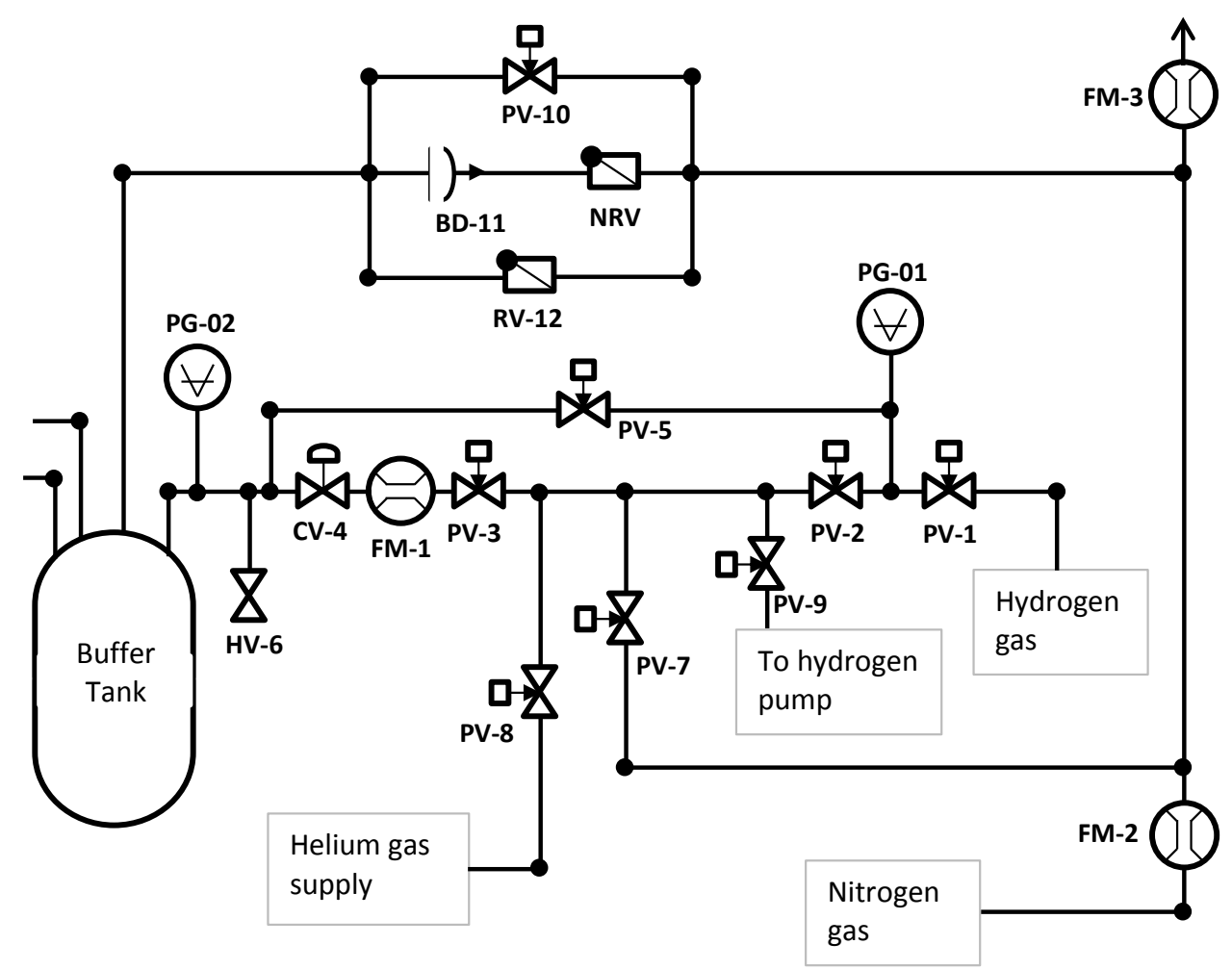

Figure 16. A schematic of the hydrogen gas panel. Key: $\mathrm{PG}=$ pressure gauge, $\mathrm{CV}=$ control valve, $\mathrm{PV}$ = process valve (normally closed), $\mathrm{HV}=$ hand-operated (manual) valve, $\mathrm{RV}=$ relief valve, $\mathrm{BD}=$ burst disc, NRV = non-return valve, and FM = flowmeter. The NRV would prevent back-streaming of nitrogen should the burst disc rupture, as this could cause blockages in the low temperature pipework. The two tubes leaving the left-hand side of the diagram connect to the transfer line.

leaked from the cold condensing unit. The high-vacuum sensor (Penning type: Leybold PTR-225) was switched off and disconnected from its controller before hydrogen was allowed into the system to avoid the possibility of high voltages being in contact with leaked hydrogen. Gas pumped from the SV was emitted into a locked and ventilated pump cabin on the roof of the experimental hall. The cabin air was extracted by fans and released to the atmosphere about $4.5 \mathrm{~m}$ above roof level. All the equipment in this cabin was ATEX-rated.

The large $(150 \mathrm{~mm})$ bore pipe from the SV to the inlet of the turbo-molecular pump formed part of the emergency quench line. If there had been a sudden rupture of the thin aluminium windows of the absorber vessel that released a large quantity of hydrogen gas into the $\mathrm{SV}$, and if the pressure in this line had exceeded atmospheric pressure, the hydrogen would have escaped through a lift-off plate situated in the pump cabin and thence been safely extracted to atmosphere.

\subsection{Gas panel}

The gas panel was situated inside an enclosure that contained the nitrogen-gas jacket. All the valves within the enclosure were actuated by compressed air which was controlled using electrical actuators attached to the exterior of the enclosure; the compressed-air tubes entered the enclosure through 
grommets. Pressure sensors were ATEX-rated (IIC) Pepperl and Fuchs 24 V DC transducers, PPCM10, for the range from vacuum to 4 bar. The flow sensor FM-1 was an Ex-Flow Bronkhorst X100 (ATEX-rated IIC).

A schematic of the hydrogen gas panel is shown in figure 16. Hydrogen gas supplied from the MCP was regulated to about 1350 mbar, as measured at PG-01; excessive pressures in this supply line were limited by a $10 \mathrm{psig}$ relief valve to atmosphere outside the building. The gas flow (transient rate and cumulative quantity) into the buffer tank, through the control valve CV-4, was measured by FM-1. This buffer tank was directly connected (no valves) to the condensing unit via the transfer line. During cooling and liquefaction, the pressure at PG-02 was automatically maintained at 1150 mbar using CV-4. The maximum pressure in the buffer tank was limited to 1500 mbar by the relief valve, RV-12, which would release excess hydrogen gas to the vent line that contained flowing nitrogen gas $(\sim 5 \mathrm{l} / \mathrm{min}$.) at atmospheric pressure. Also, the process valves PV-7 and PV-10 enabled the operator to release excess pressure into this vent line. A difference in flow rate between flowmeters FM-3 and FM-2 indicated the amount of gas being released. This nitrogen-filled vent line buffered the gas panel outlets (PV-7, PV-10, RV-12, and BD-11) from air. The burst disc was the safety back-up in case the relief valve failed to open. Valve PV-5 bypassed the flow restrictions of FM-1 and CV-4 to enable the buffer tank and condensing unit to be evacuated more readily by the hydrogen pump, and the manual valve HV-6 was used for connecting the leak detector.

\section{Control and monitoring system}

The liquid-hydrogen control and monitoring (C\&M) system was sited in a locked room adjacent to the experimental hall containing MICE. It was designed to be stand-alone, based on programmable logic controllers (PLC), and capable of operating without an external network connection to eliminate the possibility of unauthorised intervention. A touch-screen display indicated the status of the system and allowed an operator to run pre-determined sequences such as 'Purge', 'Fill', and 'Empty'. The control of individual system components (valves, pumps, compressor etc.) was also possible using this touch-screen. Keyed switches were provided to allow manual override (by authorised persons only) of certain automatic functions should this be required. The control PLC was an Omron CJ1M-CPU13-ETN PLC and the touch-screen an Omron NS8-TV01B-V2. The control cabinet contained Intrinsically Safe Barriers (Pepperl and Fuchs), Flowmeter Readout Unit (Bronkhorst), Vacuum Gauge Controller (Leybold) and the Temperature Monitors (Lakeshore 218).

A second PLC was used for the gas-detection system, which incorporated an Oliver IGD TOCSIN 920 control panel, and the extraction system. The extraction system used two $7.5 \mathrm{~kW}$ non-sparking fans in parallel, each controlled by an Omron MX2-Inverter. These fans ensured that the gas-panel enclosure was kept at a pressure below atmospheric. One fan alone was capable of maintaining the required air flow; their speeds were reduced to $50 \%$ when both fans were running. If escaped hydrogen had been detected, both fans would have automatically run at $100 \%$ to clear all this hydrogen gas as quickly as possible. The fans were fitted with differential pressure switches (RedBin-P500-2) to monitor their operation. The status of the inverters was also monitored and displayed on the NS8 touch-screen. An uninterruptable power supply (UPS: Rello MST 20-T4-1) of adequate size allowed the extraction system, in the event of a power failure, the capability to run 

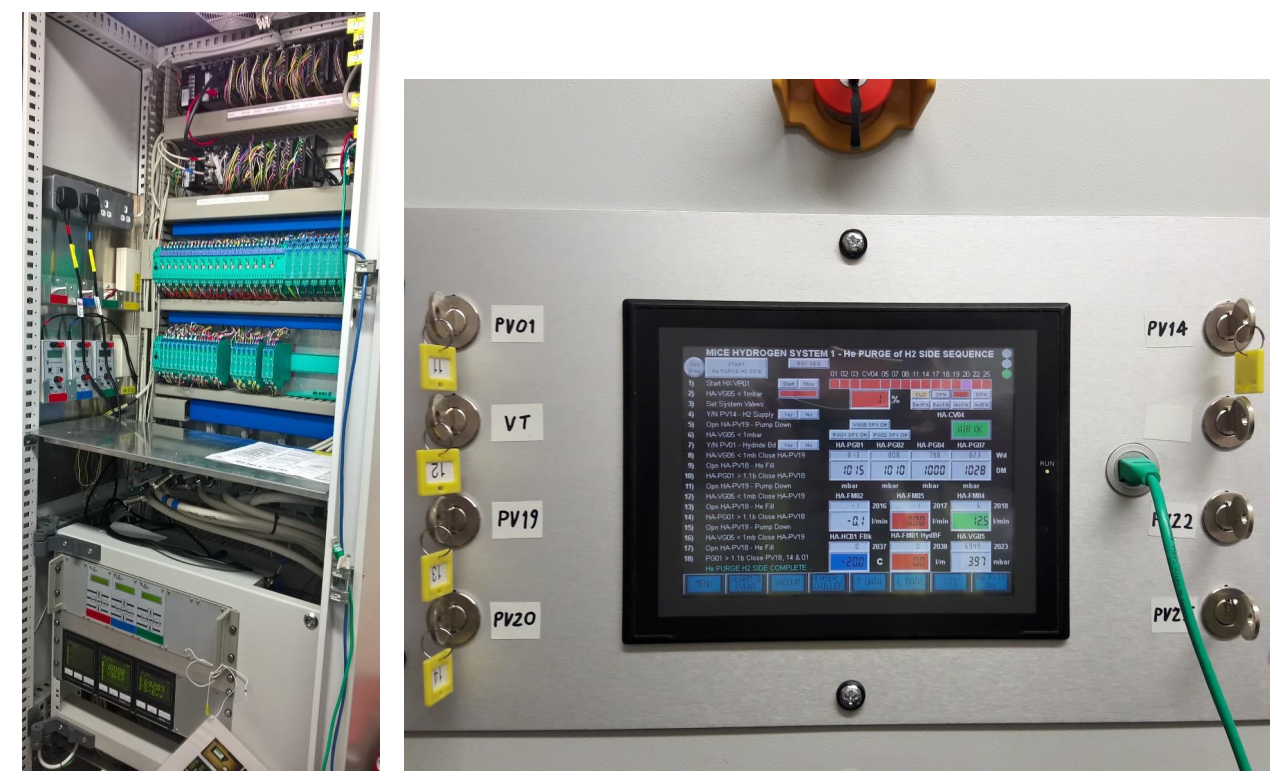

Figure 17. Photographs of the $\mathrm{H}_{2}$-system control cabinet (left), and the touch-screen panel (right) between the keyed over-ride switches and below the emergency shut-down button.

the fans for a sufficiently long period during which the entire system would have been emptied of hydrogen. The control PLC and gas-detection system were also powered by this UPS unit.

The 'intrinsically-safe' explosion-protection method was used for all equipment in the gas panel and all of the sensors in the absorber. Since the heaters attached to the absorber vessel and the coldhead could not be intrinsically safe, they were interlocked to prevent their operation unless the vacuum in the SV was better than $10^{-3}$ mbar. Layers Of Protection Analysis (LOPA) was applied to the whole control system and identified that this interlock required a safety integrity level 1 (SIL1) rating. This was achieved by providing two redundant vacuum gauges and gauge controllers to drive relays to give a dual-guard-line interlock to the heater power supply. The LOPA study also identified that a failure of the gas detection system was a hazard requiring a SIL1 rating. To achieve this, the detectors were installed in pairs on separate wiring loops.

The hydrogen system was monitored via a read-only gateway on the ethernet network and values were logged using the Experimental Physics and Industrial Control System (EPICS). Information from the Omron PLCs was retrieved using the Factory Interface Network Service (FINS) protocol which was implemented as a module in EPICS. This provided direct access to the memory registers of the PLCs and thus allowed the variables used by the PLCs to be provided as EPICS variables. Only the parameters of most interest (e.g. temperatures, pressures and valve status) were provided as EPICS variables. These were then used by the MICE Archiver and Alarm Handler in the same way as for the other MICE C\&M data. To ensure that unauthorised remote operation of the PLCs was not possible, the hydrogen-system network was isolated from the rest of the MICE and RAL site networks. A PC running EPICS was used to isolate the networks using two network cards and a network bridge providing read-only access to the EPICS variables from the MICE network only. This is shown in figure 18 . 


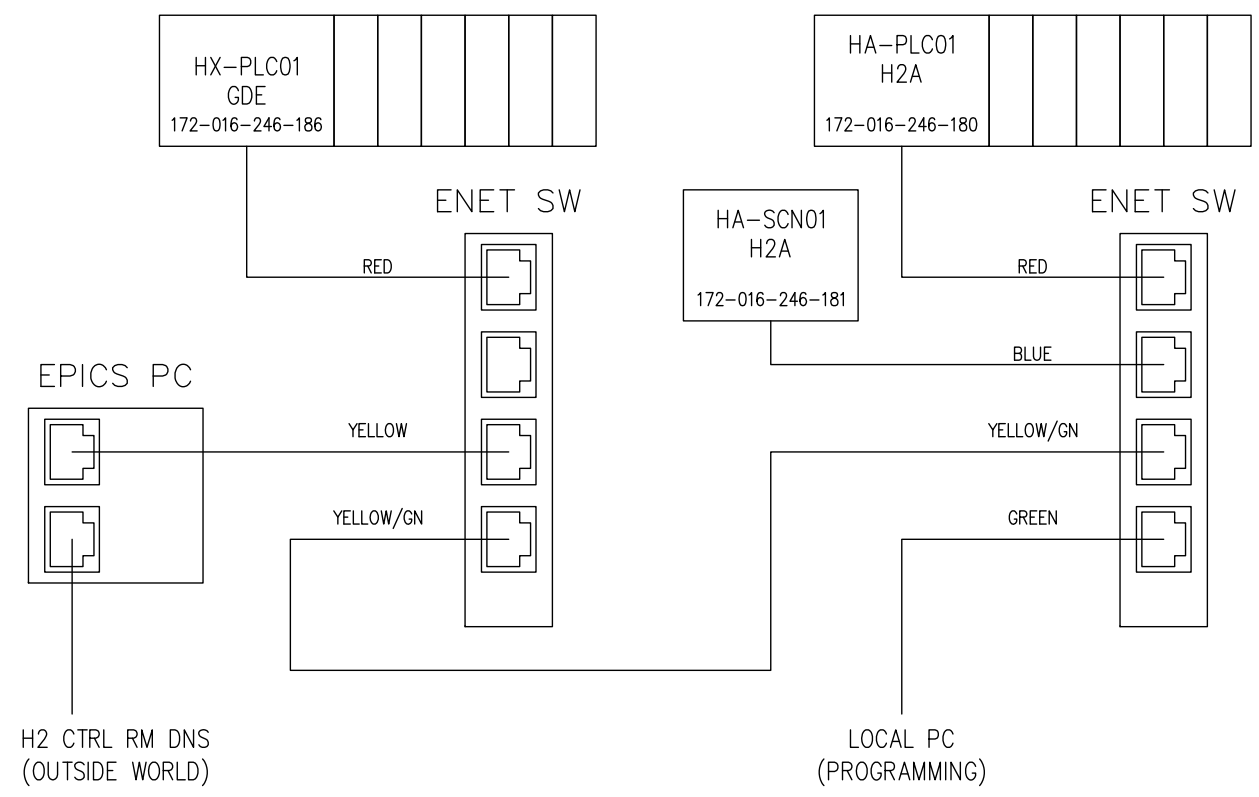

Figure 18. Diagram of the hydrogen-system network and devices: the gas-detection (GDE) PLC, and the hydrogen control system (H2A) PLC and touch-screen (SCN).

\section{Performance}

To verify the performance of the cryogenic systems and to develop and refine the control sequences, a test setup was established outside the MICE Hall. To avoid the need to establish a second hydrogen-safe gas system, the system-verification test programme was carried out using helium and neon. An identical cryostat was used in a different building to:

- Gain experience with the experimental setup;

- Fine-tune the gas circuitry;

- Implement and test any necessary changes to the pipework, thermal insulation, sensors, heaters etc.; and

- Scale the likely performance to be achieved with hydrogen in MICE.

An initial cool-down of the apparatus, using helium gas, was used to determine the base temperature of the absorber vessel. The condenser cooled the gas which preferentially passed through the lower port of the condenser and down through the thermally-insulated pipework to the bottom of the absorber vessel. The warmer gas in this absorber vessel preferentially rose through its top port and continued upwards through more thermally-insulated pipework to the top port of the condenser. These movements of gas within the system established a self-sustaining circulating gas flow. As the condenser and absorber vessel cooled, more room-temperature gas was drawn in from the supply. The incoming gas was cooled by the tubes that were heat-sunk to the thermal shield before it reached the condensing circuit. 
Table 2. Temperatures in the condenser and absorber vessel before and after modifications to the cryogenics.

\begin{tabular}{|l|c|c|}
\hline Temperatures (K) & Before modification & After modification \\
\hline Condenser With Gas & 22 & 12 \\
Condenser Evacuated & 13 & 4.5 \\
Absorber With Gas & 39.5 & 19.5 \\
\hline
\end{tabular}

\subsection{Initial tests with helium gas}

Using helium gas at a pressure of about 1150 mbar absolute, up to 12 hours were required for the gas circulation to be established and thus for the efficient cool-down of the absorber vessel to begin. The absorber vessel then cooled at a rate of about $-6 \mathrm{~K} / \mathrm{hour}$. This steady cooling of the system prevented the build-up of large thermal gradients that might have led to the development of leaks. The condenser reached $22 \mathrm{~K}$ approximately 53 hours after the cryocooler was started, and the absorber vessel reached $39.5 \mathrm{~K}$. Evacuating the gas circuit resulted in a temperature on stage two of the coldhead of $13 \mathrm{~K}$. The minimum temperature in the gas-filled condenser of $22 \mathrm{~K}$ would have been insufficient to condense hydrogen which has a boiling point of $20.3 \mathrm{~K}$ at atmospheric pressure [40]. This prompted us to improve the cryogenics of the gas circuit.

After modifications to the pipework and the thermal insulation, a second run with helium gas achieved $12 \mathrm{~K}$ on the coldhead and $19.5 \mathrm{~K}$ in the absorber vessel. And, with the gas circuit evacuated, the temperature of the cold-head dropped to $4.5 \mathrm{~K}$. This is shown in table 2 . These temperatures gave reassurance that hydrogen could now be liquefied by the system. The difference in temperatures, $\Delta T$, between the condenser and the absorber vessel was also expected to decrease when liquid collected in the absorber vessel. Figure 19 shows the performance of the cryogenic system before and after the modifications. The measurements showed that the heat-load on the condenser had been reduced from $8.75 \mathrm{~W}$ to $1.4 \mathrm{~W}$ by these modifications, and the additional heat-load from the gas-cooling of the absorber vessel had been reduced from $11 \mathrm{~W}$ to $7.5 \mathrm{~W}$.

\subsection{Liquefaction of neon}

\subsubsection{Off-line}

The next step was to use neon gas as the refrigerant in order to test the ability of the system to liquefy gas and then maintain a constant volume of liquid, and to measure the $\Delta T$ between the condenser and the absorber vessel. With neon gas flowing into the system, the temperature of the coldhead ultimately settled at $27.1 \mathrm{~K}$ and, once liquid began to collect, the absorber vessel cooled rapidly from $40 \mathrm{~K}$ to $27.8 \mathrm{~K}$ (the boiling point of neon at $\sim 120$ mbar above atmospheric pressure [40]). With neon being liquefied, $\Delta T \leq 1 \mathrm{~K}$ indicated good circulation of gas and liquid. Approximately $2 l$ of liquid neon were collected inside the absorber vessel; both the lowest sensor at the bottom of the vessel and the next-lowest sensor at $45^{\circ}$ from the vertical registered the presence of liquid. The heat of the incoming gas during the fill process, and of the boil-off gas from the absorber vessel, was sufficient to keep the condenser above the neon freezing temperature of $\sim 24.5 \mathrm{~K}$. After the external supply of gas was stopped, thus isolating the condenser/absorber circuit, the heater on the coldhead could control the system pressure above atmospheric and keep the liquid level constant. 


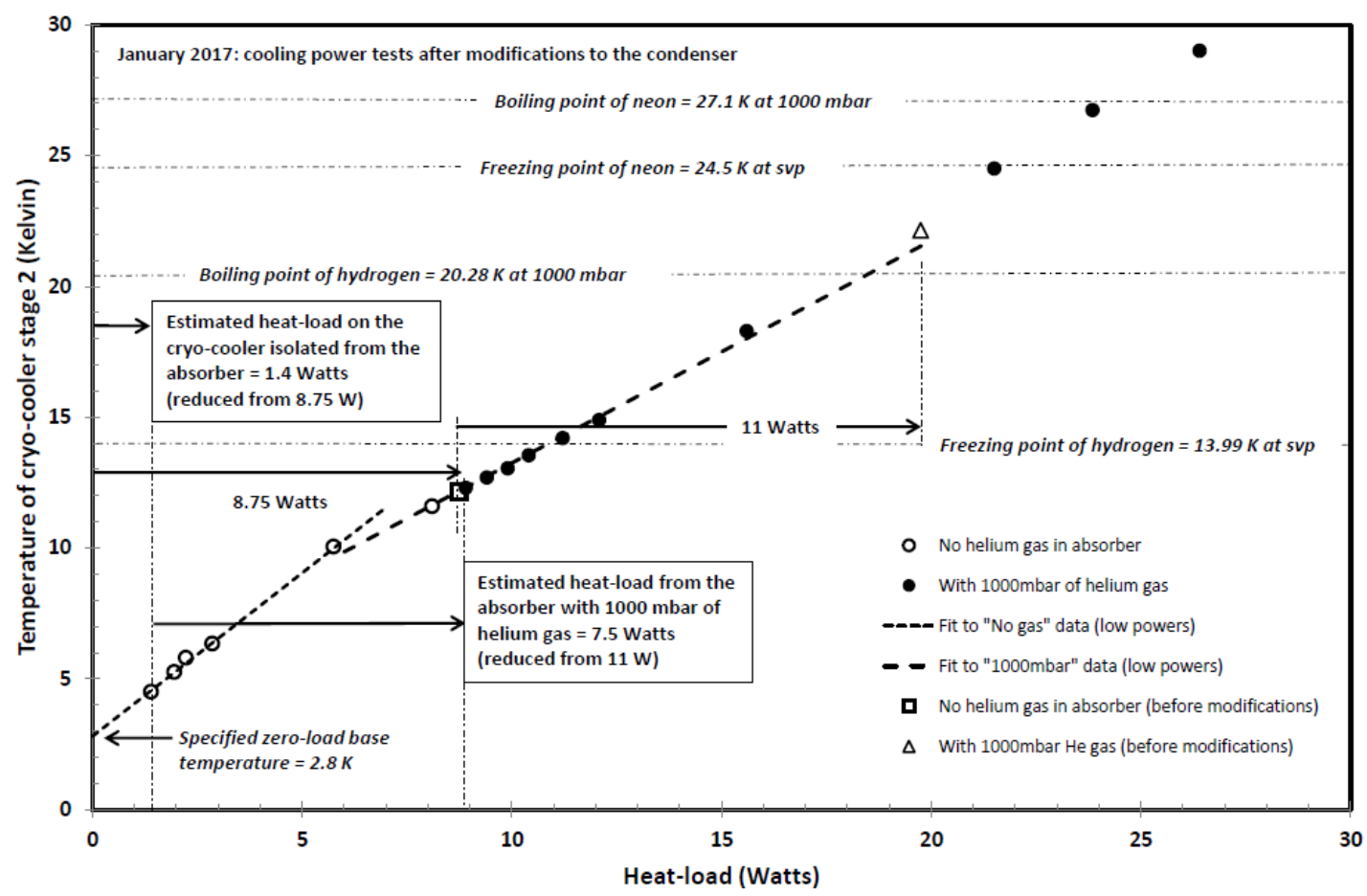

Figure 19. Cryogenic performance of the liquefaction circuit. The temperature of the second stage of the coldhead is plotted as a function of its heat-load (environmental thermal load plus Joule heating provided by an attached heater). The data with the system evacuated were translated horizontally until the intercept on the temperature axis corresponded to the specified zero-load temperature of $2.8 \mathrm{~K}$ quoted by the manufacturer of the coldhead. This estimates the environmental thermal load on the coldhead and condenser. Helium gas at a pressure of $\sim 1000$ mbar was then introduced to the condenser, and this gas cooled the absorber vessel. The gas-load data were then aligned with the vacuum data to estimate the thermal load on the absorber vessel.

This initial test demonstrated that neon gas could be condensed into the absorber, that the volume of condensed liquid could be controlled, that freezing of the liquid could be prevented via the heater on the coldhead, and that $\Delta T$ was small.

\subsubsection{Within MICE}

The cool-down and liquefaction of neon gas was successfully repeated after the system was installed within the bore of the focus-coil module in MICE. As can be seen in table 3, the energy that must be extracted to cool gas from room temperature and produce the liquid at its boiling point is less for hydrogen than it is for neon. This gave the confidence required to progress to liquefying hydrogen.

\subsection{Liquefaction of hydrogen}

The absorber vessel was pre-cooled using helium gas to transport heat between the condenser and the absorber vessel. This pre-cool lasted for four days, and ended with the following temperatures: coldhead at $44.1 \mathrm{~K}$ (first stage) and $14.0 \mathrm{~K}$ (second stage); and the absorber vessel at $21.2 \mathrm{~K}$ (top) and $20.4 \mathrm{~K}$ (bottom). After emptying the system of helium gas, hydrogen gas at $1150 \mathrm{mbar}$ was introduced. The incoming gas warmed the condenser, and the automatically-controlled heater 
Table 3. Some cryogenic data for the gases involved in this experiment. The expansion ratio is the ratio of the volume of gas to the volume of liquid it creates when condensed. $\left(T_{0}-T_{b p}\right)$ is the temperature difference between room temperature $(295 \mathrm{~K})$ and the boiling point. The bottom row gives the energy that must be extracted from room temperature gas to create the liquid at its boiling point.

\begin{tabular}{|l|c|c|c|}
\hline & Helium & Hydrogen & Neon \\
\hline Gas density $\left(\mathrm{kg} / \mathrm{m}^{3}\right)$ & 0.16 & 0.08 & 0.82 \\
Expansion ratio & 740 & 830 & 1412 \\
Mass of one liquid litre $(\mathrm{kg})$ & 0.125 & 0.071 & 1.2 \\
Specific heat capacity $c_{p}\left(\mathrm{~kJ} \mathrm{~kg}^{-1} \mathrm{~K}^{-1}\right)$ & 5.19 & 14.32 & 1.03 \\
$T_{0}-T_{b p}(\mathrm{~K})$ & 291 & 275 & 268 \\
Latent Heat $(\mathrm{kJ} / \mathrm{kg})$ & 21 & 455 & 86.3 \\
Energy per liquid litre $(\mathrm{kJ})$ & 191 & 312 & 435 \\
\hline
\end{tabular}

Cumulative condensed hydrogen gas

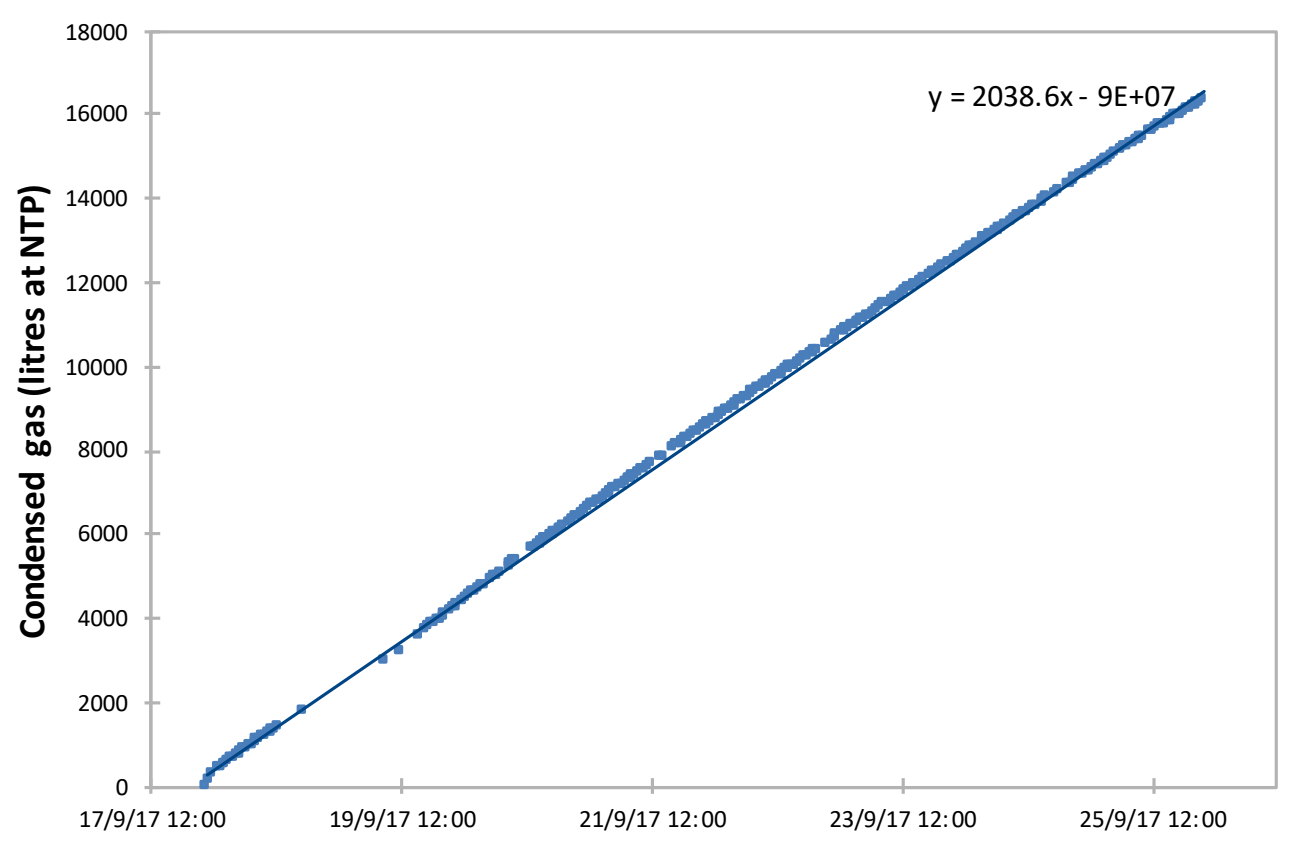

Figure 20. Filling the absorber vessel with condensing hydrogen gas. The cumulative quantity of condensed gas increased almost linearly over about 8 days, at an average rate of just over 2000 bar litres per day.

maintained the coldhead second stage temperature above $18 \mathrm{~K}$. If this temperature had decreased to below $15 \mathrm{~K}$, the control system would have automatically switched off the helium compressor that powered the coldhead to prevent freezing of the hydrogen. Final cool-down and liquefaction of hydrogen took eight days. The liquefaction rate (as measured by gas passing through FM-1) was almost uniform, as shown in figure 20. A total of $\sim 16,300$ bar $l$ of hydrogen gas were liquefied. 


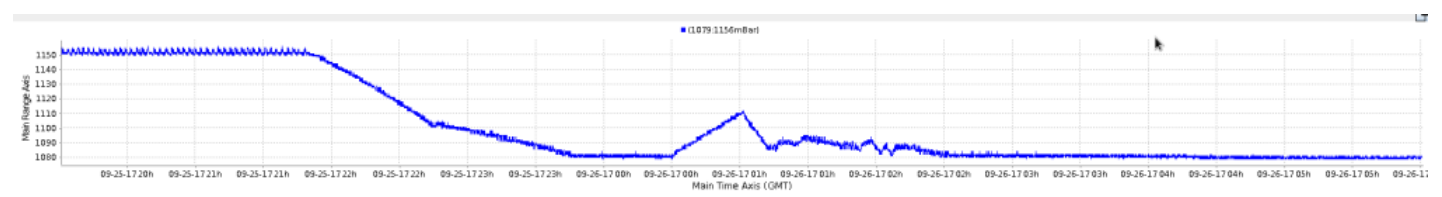

Figure 21. Pressure in the absorber vessel, as a function of time, during the change-over from the filling process ( $\mathrm{p}=1150 \mathrm{mbar}$ ) to the pressure-controlling state (1080 mbar).

Once the absorber vessel was deemed to be full (as indicated by the volume of gas condensed and the level sensors) the control sequence was changed from 'Fill' to 'Full' and the external supply of hydrogen was isolated. The operating pressure then decreased to the new setting of $1080 \mathrm{mbar}$. Figure 21 shows the pressure in the absorber vessel during this change-over. The control system then reliably maintained the pressure around this value. If the pressure had decreased below 1040 mbar, the helium compressor would have been switched off automatically.

The $22 l$ volume of liquid hydrogen was maintained for the duration of this phase of the MICE data-taking from the $25^{\text {th }}$ September 2017 to the $16^{\text {th }}$ October 2017 . The coldhead was then switched off and the heaters were switched on, delivering a nominal power of $50 \mathrm{~W}$ to the absorber vessel. The hydrogen gas vented to the atmosphere via RV-12 and the nitrogen-purged vent line. Emptying the absorber vessel took approximately 5.5 hours. The hydrogen gas was then purged from the system.

\section{Conclusions}

A complete system capable of safely condensing hydrogen gas in a vessel with thin aluminium windows was designed, constructed and operated. This vessel was placed inside the focus-coil magnet of the MICE experiment at the Rutherford Appleton Laboratory and was irradiated with a beam of muons. Approximately $22 l$ of liquid hydrogen were collected in this vessel, and this liquid was kept at a constant temperature and pressure for three weeks. This enabled the MICE collaboration to measure the loss of energy and change of trajectory of muons in liquid hydrogen, thus elucidating the details of the interactions that lead to beam-cooling effects in liquid hydrogen.

\section{Acknowledgements}

The work described here was performed to deliver the liquid-hydrogen absorber system for the international Muon Ionization Cooling Experiment built at the STFC Rutherford Appleton Laboratory (RAL) in the UK. We are indebted to the MICE collaboration for providing the motivation for, and the context within which, the work reported here was carried out. We would like to acknowledge the support and hospitality of FNAL, The Daresbury Laboratory, KEK, RAL, and the Universities of Mississippi and Oxford where designs, machining or test procedures were carried out. The work described here was made possible by grants from the Department of Energy and the National Science Foundation (USA), the Science and Technology Facilities Council (UK) and the Japan Society for the Promotion of Science. We gratefully acknowledge all sources of support. We are 
grateful for the support given to us by the staff of the STFC Rutherford Appleton and Daresbury Laboratories during the build, commissioning and operational phases of the project.

\section{References}

[1] S. Geer, "Neutrino beams from muon storage rings: characteristics and physics potential," Phys. Rev. D57 (1998) 6989 [hep-ph/9712290]

[2] M. Apollonio et al., "Oscillation physics with a neutrino factory,” [hep-ph/0210192]

[3] D. V. Neuffer and R. B. Palmer "A high-energy high-luminosity $\mu^{+}-\mu^{-}$collider," Conf. Proc. C940627 (1995) 52

[4] R. B. Palmer, “Muon colliders,” Rev. Accel. Sci. Tech. 7 (2014) 137

[5] M. Boscolo, M. Antonelli, O. R. Blanco-Garcia, S. Guiducci, S. Liuzzo, P. Raimondi, and F. Collamati, "Studies of a scheme for Low EMmittance Muon Accelerator with production from positrons on target," (2018) [physics.acc-ph/1803.06696]

[6] S. Y. Lee, Accelerator Physics (Third Edition), World Scientific Publishing Co, 2012

[7] S. Schroeder et al., "First laser cooling of relativistic ions in a storage ring," Phys. Rev. Lett. 64 (1990) 2901 [http://link.aps.org/doi/10.1103/PhysRevLett.64.2901]

[8] J. S. Hangst, M. Kristensen, J. S. Nielsen, O. Poulsen, J. P. Schiffer, and P. Shi, "Laser cooling of a stored ion beam to 1mK," Phys. Rev. Lett. 67 (1991) 1238 [http://link.aps.org/doi/10.1103/PhysRevLett.67.1238]

[9] P. J. Channell, "Laser cooling of heavy ion beams," Journal of Applied Physics 52 (1981) 3791 [http://dx.doi.org/10.1063/1.329218]

[10] J. Marriner, “Stochastic cooling overview," Nucl. Instrum. Meth. A532 (2004) 11 [physics/0308044]

[11] V. V. Parkhomchuk and A. N. Skrinsky, "Electron cooling: 35 years of development," Physics-Uspekhi 43 (2000) 433 [http: //stacks.iop.org/1063-7869/43/i=5/a=R01]

[12] A. N. Skrinsky and V. V. Parkhomchuk," Cooling methods for beams of charged particles (In Russian)," Sov. J. Part. Nucl. 12 (1981) 223

[13] D. Neuffer, "Principles and applications of muon cooling," Conf. Proc. C830811 (1983) 481

[14] D. Neuffer, "Principles and applications of muon cooling," Part. Accel. 14 (1983) 75

[15] D. Stratakis, R. C. Fernow, J. S. Berg, and R. B. Palmer, "Tapered channel for six-dimensional muon cooling towards micron-scale emittances," Phys. Rev. ST Accel. Beams 16 (2013) 091001

[16] D. Stratakis, and R. B. Palmer, "Rectilinear six-dimensional ionization cooling channel for a muon collider: a theoretical and numerical study,” Phys. Rev. ST Accel. Beams 18 (2015) 031003

[17] MICE Collaboration, "MICE: an international Muon Ionization Cooling Experiment," MICE Note 21 (2003) [http://mice.iit.edu/micenotes/public/pdf/MICE0021/MICE0021.pdf]

[18] MICE Collaboration, "MICE: an international Muon Ionization Cooling Experiment, Technical Reference Document," MICE-TRD-2005

[19] C. N. Booth et al., "The design, construction, and performance of the MICE target," JINST 8 (2013) P03006 [physics.ins-det/1211.6343] 
[20] C. N. Booth et al., “The design and performance of an improved target for MICE,” JINST 11 (2016) P05006 [physics.ins-det/1603.07143]

[21] M. Bogomilov et al., "The MICE muon beam on ISIS and the beam-line instrumentation of the Muon Ionization Cooling Experiment,” JINST 7 (2012) P05009 [physics . acc-ph/1203 . 4089]

[22] D. Adams et al., "Characterisation of the muon beams for the Muon Ionization Cooling Experiment," Eur. Phys. J. C73 (2013) 2582 [physics.acc-ph/1306. 1509]

[23] R. Bertoni et al., "The design and commissioning of the MICE upstream time-of-flight system," Nucl. Instrum. Meth. A615 (2010) 14 [physics.ins-det/1001 . 4426]

[24] R. Bertoni, M. Bonesini, A. de Bari, G. Cecchet, Y. Karadzhov, and R. Mazza, "The construction of the MICE TOF2 detector," (2010) [http://mice.iit.edu/micenotes/public/pdf/MICE0286/MICE0286.pdf]

[25] L. Cremaldi, D. A. Sanders, P. Sonnek, D. J. Summers, and J. Reidy, Jr, "A Cherenkov radiation detector with high density aerogels,” IEEE Trans. Nucl. Sci. 56 (2009) 1475 [physics.inst-det/0905.3411]

[26] M. Ellis et al., "The design, construction, and performance of the MICE scintillating fibre trackers," Nucl. Instrum. Meth. A659 (2011) 136 [physics.ins-det/1005. 3491]

[27] F. Ambrosino et al., "Calibration and performance of the KLOE calorimeter," Nucl. Instrum. Met. A598 (2009) 239

[28] R. Asfandiyarov et al., "The design and construction of the MICE electron-muon ranger," JINST 11 (2016) T10007 [physics.ins-det/1607.04955]

[29] A. Dobbs, C. Hunt, K. Long, E. Santos, M. A. Uchida, P. Kyberd, C. Heidt, S. Blot, and E. Overton, “The reconstruction software for the MICE scintillating fibre trackers," JINST 11 (2016) T12001 [physics.inst-det/1610.05161]

[30] V. Blackmore, J. Cobb, M. Dawson, J. Tacon, and M. Tacon, "Particle tracking and beam matching through the new variable thickness MICE diffuser," Conf. Proc. C110328 (2011) 154

[31] “Explosive atmospheres CompEx Ex F foundation course,” ExVeritas, Wrexham UK (2015)

[32] “SA/SMS/P3986 - HAZOP report,” SERCO Assurance, Cheshire UK (2006)

[33] “X589 TR001-2.0,” Functional Safety Consultants Ltd, UK (2010)

[34] “SF0005355 - HAZOP report,” AMEC, Warrington UK (2012)

[35] “10492-900-02 - HAZOP report,” Rowan House Ltd, Cheltenham UK (2017)

[36] Particle Data Group, M. Tanabashi et al., “The review of particle physics,” Physical Review D 98 (2018) 030001

[37] D. Kaplan, E. Black, K. Cassel, and M. Cummings, “Energy absorber R\&D,” Nucl. Instrum. Meth. A472 (2001) 632

[38] S. Ishimoto et al., “Convection-type $\mathrm{LH}_{2}$ absorber $\mathrm{R} \& D$ for muon ionization cooling," Nucl. Instrum. Meth. A503 (2003) 396

[39] M. Cummings et al., “Current $\mathrm{LH}_{2}$-absorber $\mathrm{R} \& D$ in MuCool,” Journal of Physics G: Nuclear and Particle Physics,” 29 (2003) 1689

[40] National Institute of Standards and Technology, US Dept. of Commerce, "NIST chemistry webbook," (2017) [https://webbook.nist.gov/chemistry/] 